\title{
MINKOWSKI ALGEBRA I: A CONVOLUTION THEORY OF CLOSED CONVEX SETS AND RELATIVELY OPEN CONVEX SETS*
}

\author{
BEIFANG $\mathrm{CHEN}^{\dagger}$
}

\begin{abstract}
This is the first one of a series of papers on Minkowski algebra. One of purposes of this paper is to set up a general framework so that the mixed volume theory and integral geometry can be developed algebraically in subsequent papers. The so called Minkowski algebra of convex sets is the vector space generated by indicator functions of closed convex sets and relatively open convex sets, where the multiplication is induced by the Minkowski sum of convex bodies. We shall study the homomorphisms induced by the linear maps from the ground vector spaces; the subalgebra spanned by the indicate functions of closed convex sets; the subalgebra spanned by the indicator functions of relatively open convex sets; and the embedding of these subalgebras in the commutative algebra of multivalued functions with multiplicities. With this embedding, we are able to define the Minkowski product for indicator functions of a closed convex set and a relatively open convex set, which could be technically difficult without the embedding; and to classify the multiplicative units of the algebra. Moreover, the group of units with Euler characteristic equal to +1 can be viewed as a vector space isomorphic to the vector space spanned by certain continuous functions, including support functions of convex bodies. Finally, we introduce the Euler-Radon transform for convex chains with respect to the Euler measure, and show that it is injective. This injectivity solves the syzygy problem of [10] on affine Grassmannians.
\end{abstract}

1. Introduction. Let $\mathbf{V}$ be a finite dimensional real vector space. By a convex body of $\mathbf{V}$ we mean a non-empty compact convex set. The dilation of a convex body $K$ by a non-negative real number $\lambda$ is the set $\lambda K=\{\lambda x \mid x \in K\}$. The Minkowski sum of convex bodies $K$ and $L$ is the set $K+L=\{x+y \mid x \in K, y \in L\}$. Obviously, $\lambda K$ and $K+L$ are convex bodies. With these set operations, the class of convex bodies of $\mathbf{V}$ forms a convex cone. It is well-known that the smallest vector space which contains this convex cone can be identified to the vector space generated by the support functions of convex bodies. However, for the support function $h_{K}$ of a convex body $K$, the geometric interpretation of the function $-h_{K}$ is not clear in the literature.

Finite unions of convex bodies can be of many other geometric objects such as piecewise linear manifolds and polyhedra. We shall be interested in constructing a vector space which contains at least finite unions of closed convex sets and finite unions of relatively open convex sets, and the Minkowski sum of which can be extended to a commutative multiplication. We believe the best candidate for this space is the vector space $\mathbf{R}(\mathbf{V}, \mathcal{C} \cup \mathcal{O}$ ) (see below) spanned by indicator functions of closed convex sets and relatively open convex sets. The purpose of this paper is to set up a natural framework so that the theory of mixed volumes and integral geometry can be developed algebraically.

The class of finite unions of convex bodies, called convex ring, was first considered by Hadwiger [9]. This class can not be a vector space and there is no "nice" algebraic structures. The first vector space which contains convex bodies is perhaps the vector space spanned by their support functions, that is, the functions of the form $h_{A}-h_{B}$, where $h_{A}$ and $h_{B}$ are the support functions of convex bodies $A$ and $B$ respectively. This space is isomorphic to the vector space generated by the ordered pairs of convex bodies, of which two ordered pairs $(A, B)$ and $(C, D)$ are assumed to be equal if

*Received March 24, 1998; accepted for publication December 21, 1998.

†Department of Mathematics, Hong Kong University of Science and Technology, Clear Water Bay, Kowloon, Hong Kong (mabfchen@uxmail.ust.hk). Research is supported by RGC Competitive Earmarked Research Grants HKUST595/94P and HKUST707/96P. 
$A+D=B+C$. The addition, scalar product, and difference for the pairs of convex bodies are defined by

$$
\begin{aligned}
(A, B)+(C, D) & =(A+C, B+D), \\
\lambda(A, B) & =(\lambda A, \lambda B), \\
(A, B)-(C, D) & =(A+D, B+C) .
\end{aligned}
$$

A convex body $A$ is identified to the pair $(A,\{o\})$, where $o$ is the zero vector of $\mathbf{V}$. A pair $(A, B)$ of convex bodies is identified to the difference $h_{A}-h_{B}$ of their support functions [1] [25]. Another vector space which contains convex bodies was constructed by Groemer [8] by considering linear combinations of indicator functions of convex bodies. With the inclusion-exclusion principle, the space of Groemer contains finite unions of convex bodies, and the Minkowski sum is extended to a commutative multiplication on that vector space. These two vector spaces of convex bodies are quite different: on the one hand for a convex body $K$, the function $-h_{K}$ can not be identified to any linear combination of indicator functions of convex bodies; on the other hand the indicator function of an arbitrary finite union of convex bodies can not be realized by the difference of support functions of two convex bodies.

After careful observation it was found that for a convex body $K$, the function $-h_{K}$ could be identified to the relatively open convex set -ri $K$ with positive or negative sign, where ri $K$ is the relative interior of $K$, that is, the interior of $K$ in the flat that $K$ spans. This shows that the class of relatively open convex sets has to be considered in order to have Minkowski multiplicative inverse for convex bodies. We now study the vector space $\mathbf{R}(\mathbf{V}, \mathcal{C} \cup \mathcal{O})$ over the field $\mathbf{R}$ of real numbers.

Following the idea of Groemer [8] and making use of the Euler characteristics in [3] [24], the Minkowski sum of convex bodies can be extended to a commutative binary operation on the vector space spanned by indicator functions of closed convex sets and on the vector space spanned by indicator functions of relatively open convex sets. However, the difficulty arises when considering the Minkowski multiplication of the indicator functions of a closed convex set and a relatively open convex set, since the intersection of a closed convex set and a relatively open convex set is irregular in the sense that it is neither closed nor relatively open. The intersection of the boundary part could be complicated [12]. For example, if $K_{1}$ is the convex hull of the set $\left\{e^{i \theta_{2 k}} \mid k>0\right\}$ and $K_{2}$ is the convex hull of $\left\{e^{i \theta_{2 k+1}} \mid k \geq 0\right\}$ in the complex plane $\mathbf{C} \simeq \mathbf{R}^{2}$, where $\theta_{k}$ is a strictly increasing sequence of real numbers convergent to $2 \pi$, then there are infinitely many open line segments on the boundary of $K_{1} \cap$ ri $K_{2}$.

In this paper, we will avoid this irregularity through the embedding of $\mathbf{R}(\mathbf{V}, \mathcal{C} \cup \mathcal{O})$ in a commutative algebra so that the Minkowski multiplication can be replaced by the multiplication of that algebra. Thus the Minkowski multiplication of the indicator functions of a closed convex set and a relatively open convex set can be well-defined. Moreover, with this embedding a subgroup of multiplicative units with the Euler characteristic equal to +1 can be viewed as a vector space isomorphic to the vector space spanned by support functions of convex bodies.

The extension of the Minkowski sum to unbounded closed convex sets and to relatively open convex sets was once considered by Lawrence [13]. The Minkowski product considered in [13] depends unnaturally on the dimension of the ground vector space. There are two Minkowski multiplications constructed by the author in [4], one of which was missed in [13], due to lack of the Euler characteristic $\bar{\chi}$. A right extension of the Minkowski sum to relatively open convex setis and to adhesive closed convex sets [11] was obtained by Przeslawski [17], but the method there cannot be applied to 
arbitrary unbounded closed convex sets. The Minkowski algebra of polyopes had been applied in [2] to obtain numerous results on curvatures of polyhedral spaces. Morelli [15], Pukhlikov and Khovanskii [18] also considered the extension of the Minkowski sum of polytopes to polyhedra. But the most general case for closed convex sets and relatively open convex sets was the setting in [4]. A dual form of the Minkowski product is the Geissinger multiplication [5] [7], and the mixed volume of convex bodies can be naturally written as, up to a multiple constant, the volume of the Geissinger product of the indicator functions of the given convex bodies [5].

Throughout this paper, we use $\mathbf{V}^{*}$ to denote the dual vector space of $\mathbf{V}$, that is, the space of all linear functionals on $\mathbf{V}$. For a convex subset $E$ of $\mathbf{V}$, the indicator function of $E$ is the characteristic function $1_{E}$ on $\mathrm{V}$, defined by $1_{E}(x)=1$ for $x \in E$ and $1_{E}(x)=0$ otherwise. The positive support function of $E$ is the map $h_{E}^{+}: \mathrm{V}^{*} \longrightarrow$ $\overline{\mathbf{R}}$, defined by

$$
h^{+}(E, \ell)=h_{E}^{+}(\ell)=\sup \{\ell(x) \mid x \in E\}, \quad \forall \ell \in \mathbf{V}^{*},
$$

where $\overline{\mathbf{R}}=\mathbf{R} \cup\{ \pm \infty\}$. The negative support function of $E$ is the map $h_{E}^{-}: \mathbf{V}^{*} \longrightarrow \overline{\mathbf{R}}$, defined by

$$
h^{-}(E, \ell)=h_{E}^{-}(\ell)=\inf \{\ell(x) \mid x \in E\}, \quad \forall \ell \in \mathbf{V}^{*} .
$$

The notation can be justified by $h_{E}^{-}(\ell)=-h_{E}^{+}(-\ell)=-h_{-E}^{+}(\ell), \ell \in \mathbf{V}^{*}$. The positive support function $h_{E}^{+}$is the usual support function $h_{E}$ in the literature. For a family $\mathcal{E}$ of subsets of $\mathbf{V}$, we denote by $\mathbf{R}(\mathbf{V}, \mathcal{E})$ the vector space spanned by indicator functions of subsets of $\mathcal{E}$, that is, each member $f \in \mathbf{R}(\mathbf{V}, \mathcal{E})$ is a linear combination

$$
f=a_{1} 1_{E_{1}}+\cdots+a_{m} 1_{E_{m}},
$$

where $E_{i} \in \mathcal{E}, a_{i} \in \mathbf{R}, i=1,2, \cdots, m$. The subset of functions of $\mathbf{R}(\mathbf{V}, \mathcal{E})$ with integer coefficients in the linear combination is denoted by $\mathbf{Z}(\mathbf{V}, \mathcal{E})$. We shall study such vector spaces where $\mathcal{E}$ takes one of the following classes of subsets:

$\mathcal{C}=$ the class of closed convex sets,

$\mathcal{O}=$ the class of relatively open convex sets,

$\mathcal{C}_{b}=$ the class of bounded closed (compact) convex sets,

$\mathcal{O}_{b}=$ the class of bounded relatively open convex sets,

$\mathcal{P}=$ the class of convex polyhedra.

By a polyhedron we mean a subset of $\mathrm{V}$ which can be obtained by taking unions, intersections, and complements finitely many times of half-spaces. Thus a polyhedron is an element of the Boolean algebra generated by half-spaces. The elements of $\mathbf{R}(\mathbf{V}, \mathcal{C} \cup \mathcal{O})$ are called convex chains. For elementary properties of convex bodies, the reader is referred to [19] [25].

2. Euler Measures. For fixed real numbers $r$ and $s$ such that $r+s=1$ and for a real-valued function $f(x)$ on $\mathbf{R}$, the Euler integral of $f$ is defined by

$$
\chi(f)=\int f(x) \mathrm{d} \chi(x)=\sum_{x \in \overline{\mathbf{R}}}\left[f(x)-r f\left(x^{-}\right)-s f\left(x^{+}\right)\right],
$$

where $f\left(x^{-}\right)$and $f\left(x^{+}\right)$are the left and right limits of $f$ at $x \in \overline{\mathbf{R}}$ respectively, and we assume that $f\left(+\infty^{+}\right)=f\left(-\infty^{-}\right)=0, f\left(+\infty^{-}\right)=\lim _{x \rightarrow+\infty} f(x), f\left(-\infty^{+}\right)=$ $\lim _{x \rightarrow-\infty} f(x)$. The function $f$ is said to be Euler integrable if $f$ is continuous except 
at finitely many points and has both left and right limits at those discontinuous points, including positive and negative infinities.

Let $\mathbf{V}$ be an $n$-dimensional real vector space and let $\mathbf{b}=\left\{v_{1}, \cdots, v_{n}\right\}$ be a basis of V. A function $g$ on $\mathbf{V}$ can be represented as a function $g_{\mathbf{b}}\left(x_{1}, \cdots, x_{n}\right)$ of $n$ coordinate variables $x=\left(x_{1}, \cdots, x_{n}\right)$. The Euler integral of $g$ with respect to the basis $\mathbf{b}$ is defined by the iterated Euler integral

$$
\chi(g)=\int_{\mathbf{V}} g(x) \mathrm{d} \chi(x)=\underbrace{\int \cdots \int}_{n} g_{\mathbf{b}}\left(x_{1}, \cdots, x_{n}\right) \mathrm{d} \chi\left(x_{1}\right) \cdots \mathrm{d} \chi\left(x_{n}\right) .
$$

A function on $\mathbf{V}$ is said to be Euler integrable with respect to the basis $\mathbf{b}$ if each of its iterated Euler integrals is Euler integrable, and a function is simply said to be Euler integrable if it is so under all bases. When $g$ is an indicator function of a set $E$, we write $\chi(E)=\chi(g)$, and it is called Euler measure, or Euler characteristic, of $E$, since $\chi$ is actually a finitely additive set function on finite unions of closed convex sets and on finite unions of relatively open convex sets [3]. The advantage of the integral definition (2) for Euler characteristic is that we need not to consider the extension of measures, and that the Fubini theorem obviously holds.

It is clear that the set of Euler integrable functions on $\mathbf{V}$ is a subspace of realvalued functions on $\mathbf{V}$. It has been shown that the indicator function of a relatively open convex set, or a closed convex set, is Euler integrable. More precisely, if $U$ is a relatively open convex set and $F$ a closed convex set of $\mathbf{V}$, it has been shown in [3] that

$$
\begin{aligned}
& \chi(U)=(-1)^{\operatorname{dim} U}, \\
& \chi(F)= \begin{cases}(-1)^{\operatorname{dim} W} & \text { if } F=K \oplus W \\
0 & \text { if } F=M \oplus W \oplus L,\end{cases}
\end{aligned}
$$

where $K$ is a compact convex set, $W$ is a subspace, called the linearity of $F, M$ is a manifold with or without boundary, $L$ is a closed half-line, and $\oplus$ means direct sum, that is, $u \in E_{1} \oplus E_{2}$ if and only if there exist unique $u_{1} \in E_{1}$ and $u_{2} \in E_{2}$ such that $u=u_{1}+u_{2}$. (4) is called the direct sum decomposition of $F$.

One can define another Euler measure $\bar{\chi}$ for closed polyconvex sets ( $=$ finite unions of closed convex sets) and relatively open polyconvex sets (= finite unions of relatively open convex sets). Let $E$ be either a closed or a relatively open polyconvex set. We define

$$
\bar{\chi}(E)=\lim _{\rho \rightarrow+\infty} \chi[E \cap \operatorname{cl} B(o, \rho)],
$$

where $B(o, \rho)$ is the open ball of radius $\rho$ centered at the origin $o$ and $\operatorname{cl} B(o, \rho)$ is the closure of $B(o, \rho)$. To avoid the situation of intersection of a closed convex set and a relatively open convex set (the intersection may be a discrete set of infinite singletons), one may replace $B(o, \rho)$ by the open $n$-cube $I_{\rho}^{n}=(-\rho, \rho)^{n}$ if it is necessary. We have shown in [3] that if $F$ is a closed convex set and $U$ a relatively open convex set, then

$$
\begin{aligned}
& \bar{\chi}(F)=1, \\
& \bar{\chi}(U)= \begin{cases}(-1)^{\operatorname{dim} D} & \text { if } U=D \oplus W \\
0 & \text { if } U=\operatorname{ri} M \oplus W \oplus \operatorname{ri} L,\end{cases}
\end{aligned}
$$


where $D$ is a bounded relatively open convex set, $W$ is a subspace, called the linearity of $U$, ri $M$ is a manifold without boundary, and ri $L$ is an open half-line. (6) is called the direct sum decomposition of $U$.

One can also define the Euler measure $\bar{\chi}$ for closed and relatively open convex sets by making use of the same Euler integral (2), but interpreting the indicator functions of a closed convex set and a relatively open convex set on the vector space $\mathbf{V}$ over $\overline{\mathbf{R}}$ in a proper way in each direction at infinity. For instance, we can set

$$
\begin{aligned}
& 1_{\tilde{U}}( \pm \infty v)=\lim _{\rho \rightarrow \pm \infty} 1_{U}(\rho v), \quad v \in \mathbf{V}, \\
& 1_{\tilde{F}}(+\infty v)=\lim _{\substack{u \in F, \theta(u, v) \rightarrow 0 \\
\|u\| \rightarrow+\infty}} 1_{F}(u), \quad v \in \mathbf{V},
\end{aligned}
$$

where $\theta(u, v)$ denotes the angle between $u$ and $v$, and $1_{\tilde{F}}(-\infty v)=1_{\tilde{F}}(+\infty(-v))$ for any non-zero vector $v$. Then $\bar{\chi}(U)$ and $\bar{\chi}(F)$ are the Euler integrals of $1_{\tilde{U}}$ and $1_{\bar{F}}$ respectively.

The convex sets, either closed or relatively open, with a half-line direct sum decomposition in (4) and (6) are called type $I I$ sets. All other convex sets are called type $I$ sets. Given a convex set $E$, we define its recession cone $\operatorname{rec} E$ by

$$
\operatorname{rec} E=\{v \in \mathbf{V} \mid \exists p \in E \text { such that } p+t v \in E, t \geq 0\}
$$

The recession cone rec $E$ can be characterized as the set of vectors $v$ of $\mathbf{V}$ such that $v+E \subset E$. Notice that $\operatorname{rec} E$ is always a closed convex cone and $\operatorname{rec}(\operatorname{cl} E)=\operatorname{rec}(\operatorname{ri} E)$. Intuitively, $\operatorname{rec} E$ can be viewed as the cone of $E$ at infinity. For $x \notin E$, we denote

$$
x E=\{v \in \mathbf{V} \mid \exists y \in E, t>0 \text { such that } v=t(y-x)\} .
$$

If $E$ is translated to the origin $o$ by one $x \in E$, then the largest subspace spanned by $E-x$, denoted by $\langle E\rangle$, is independent of the choice of $x \in E$.

3. Induced Linear Maps. Let $\mathbf{V}$ and $\mathbf{W}$ be finite dimensional real vector spaces and $\phi$ an affine linear map from $\mathbf{V}$ to $\mathbf{W}$. $\phi$ induces a natural linear map $\phi^{*}$ from $\mathbf{R}(\mathbf{W}, \mathcal{C} \cup \mathcal{O})$ to $\mathbf{R}(\mathbf{V}, \mathcal{C} \cup \mathcal{O})$, defined by the pullback

$$
\phi^{*}(f)=f \circ \phi, \quad \forall f \in \mathbf{R}(\mathbf{W}, \mathcal{C} \cup \mathcal{O}) .
$$

We are interested in the construction of push-forward maps induced by $\phi$, since this usually cannot be done for arbitrary functions. However, there are four nontrivial push-forward linear maps $\phi_{*}^{\chi}, \bar{\phi}_{*}^{\chi}, \phi_{*}^{\bar{\chi}}, \bar{\phi}_{*}^{\bar{\chi}}$ from $\mathbf{R}(\mathbf{V}, \mathcal{C} \cup \mathcal{O})$ to $\mathbf{R}(\mathbf{W}, \mathcal{C} \cup \mathcal{O})$, defined by

$$
\begin{aligned}
& \phi_{*}^{\chi}(f)(w)=\lim _{\rho \rightarrow 0} \chi\left(f \cdot 1_{\phi^{-1}[c l B(w, \rho)]}\right), \\
& \bar{\phi}_{*}^{\chi}(f)(w)=\lim _{\rho \rightarrow 0} \chi\left(f \cdot 1_{\phi^{-1}[B(w, \rho)]}\right), \\
& \phi_{*}^{\bar{\chi}}(f)(w)=\lim _{\rho \rightarrow 0} \bar{\chi}\left(f \cdot 1_{\phi^{-1}[c l B(w, \rho)]}\right), \\
& \bar{\phi}_{*}^{\bar{\chi}}(f)(w)=\lim _{\rho \rightarrow 0} \bar{\chi}\left(f \cdot 1_{\phi^{-1}[B(w, \rho)]}\right),
\end{aligned}
$$

where $f \in \mathbf{R}(\mathbf{V}, \mathcal{C} \cup \mathcal{O}), w \in \mathbf{W}, B(w, \rho)$ is the open ball of radius $\rho$ in $\mathbf{W}$, centered at $w$, and $\operatorname{cl} B(w, \rho)$ is the closure of $B(w, \rho)$. Before we give explicit formulas for $\phi_{*}^{\chi}$, 
$\bar{\phi}_{*}^{\chi}, \phi_{*}^{\bar{\chi}}, \bar{\phi}_{*}^{\bar{\chi}}$, we prove the following lemma which will be needed often in the proof of later theorems.

LEMMA 3.1. Let $\mathrm{V}$ and $\mathrm{W}$ be finite dimensional real vector spaces and $\phi$ an affine linear map from $\mathbf{V}$ to $\mathrm{W}$. If $E$ is a convex set of $\mathrm{V}$, either closed or relatively open, then for all $w \in \phi(E), \phi^{-1}(w) \cap E$ are simultaneously type I sets or simultaneously type II sets.

Proof. It follows from the affine property of $\phi$ that there exists a linear map $f$ from $\mathrm{V}$ to $\mathbf{W}$ such that $\phi(v)=\phi(o)+f(v), v \in \mathbf{V}$. Then

$$
\phi^{-1}(w) \cap E=f^{-1}(w-\phi(o)) \cap E
$$

are parallel convex sets for all $w \in \phi(E)$. Let $w_{1}, w_{2} \in \phi(E)$. Assume that $\phi^{-1}\left(w_{1}\right) \cap E$ is a type II set, say $\phi^{-1}\left(w_{1}\right) \cap E=K_{1} \oplus M_{1} \oplus L_{1}$, where $K_{1}$ is a bounded convex set, $M_{1}$ is a manifold with or without boundary, and $L_{1}$ is a half-line. Let $v_{1} \in$ $\phi^{-1}\left(w_{1}\right) \cap E$ and $v_{2} \in \phi^{-1}\left(w_{2}\right) \cap E$. Note that $\phi\left(v_{1}\right)=\phi(o)+f\left(v_{1}\right)=w_{1}$ and $\phi\left(v_{1}+L_{1}\right)=\phi(o)+f\left(v_{1}+L_{1}\right)=\phi(o)+f\left(v_{1}\right)+f\left(L_{1}\right)=w_{1}$. Then $f\left(L_{1}\right)=o$. Thus $\phi\left(v_{2}+L_{1}\right)=\phi(o)+f\left(v_{2}\right)=\phi\left(v_{2}\right)=w_{2}$. This shows that $v_{2}+L_{1}$ is contained in $\phi^{-1}\left(w_{2}\right) \cap E$. On the other hand, if $v_{2}-L_{1}$ is contained in $\phi^{-1}\left(w_{2}\right) \cap E$, then with the same argument $v_{1}-L_{1}$ should be contained in $\phi^{-1}\left(w_{1}\right) \cap E$, a contradiction to the half-line direct surn decomposition of $\phi^{-1}\left(w_{i}\right) \cap E$. Therefore $\phi^{-1}\left(w_{2}\right) \cap E$ is a type II set.

Let $U$ be a relatively open convex set and let $F$ a closed convex set of $\mathbf{V}$. By definition we have for all $w \in \mathbf{W}$,

$$
\begin{aligned}
& \phi_{*}^{\chi}\left(1_{U}\right)(w)=\lim _{\delta \rightarrow 0} \chi\left(U \cap \phi^{-1}[\operatorname{cl} B(w, \delta)]\right)=\chi\left(U \cap \phi^{-1}(w)\right), \\
& \phi_{*}^{\chi}\left(1_{F}\right)(w)=\lim _{\delta \rightarrow 0} \chi\left(F \cap \phi^{-1}[\operatorname{cl} B(w, \delta)]\right)=\chi\left(F \cap \phi^{-1}(w)\right), \\
& \phi_{*}^{\bar{\chi}}\left(1_{U}\right)(w)=\lim _{\delta \rightarrow 0} \bar{\chi}\left(U \cap \phi^{-1}[\operatorname{cl} B(w, \delta)]\right)=\bar{\chi}\left(U \cap \phi^{-1}(w)\right) .
\end{aligned}
$$

By making use of the affine property of $\phi$, one can easily check the following

$$
\begin{aligned}
& \phi_{*}^{\chi}\left(1_{U}\right)=(-1)^{\operatorname{dim} U-\operatorname{dim} \phi(U)} 1_{\phi(U)}, \\
& \bar{\phi}_{*}^{\chi}\left(1_{U}\right)=(-1)^{\operatorname{dim} U} 1_{c l}[\phi(U)] \\
& \phi_{*}^{\bar{\chi}}\left(1_{F}\right)=1_{\mathrm{cl}[\phi(F)]}, \\
& \bar{\phi}_{*}^{\bar{\chi}}\left(1_{F}\right)=(-1)^{\operatorname{dim} \phi(F)} 1_{\mathrm{ri}[\phi(F)]} .
\end{aligned}
$$

Now if the fiber $U \cap \phi^{-1}(w)$ for one $w \in \phi(U)$ is a type II set, then by (6) and the affine property of $\phi$,

$$
\phi_{*}^{\bar{\chi}}\left(1_{U}\right)=\bar{\phi}_{*}^{\bar{\chi}}\left(1_{U}\right)=0
$$

while if the fiber $U \cap \phi^{-1}(w)$ for all $w \in \phi(U)$ are type I sets, then by (6) and the affine property of $\phi$,

$$
\begin{aligned}
& \phi_{*}^{\bar{\chi}}\left(1_{U}\right)=(-1)^{\operatorname{dim} U-\operatorname{dim} \phi(U)+\operatorname{dim} H-\operatorname{dim} \phi(H)} 1_{\phi(U)}, \\
& \bar{\phi}_{*}^{\bar{\chi}}\left(1_{U}\right)=(-1)^{\operatorname{dim} U+\operatorname{dim} H-\operatorname{dim} \phi(H)} 1_{\mathrm{cl}[\phi(U)]},
\end{aligned}
$$

where $H$ is a maximal affine subspace contained in $U$. 
Similarly, if the fiber $F \cap \phi^{-1}(w)$ for one $w \in \phi(F)$ is a type II set, then by (4) and the affine property of $\phi$,

$$
\phi_{*}^{\chi}\left(1_{F}\right)=\bar{\phi}_{*}^{\chi}\left(1_{F}\right)=0
$$

while if the fiber $F \cap \phi^{-1}(w)$ for all $w \in \phi(F)$ are type I sets, then by (4) and the affine property of $\phi$,

$$
\begin{aligned}
& \phi_{*}^{\chi}\left(1_{F}\right)=(-1)^{\operatorname{dim} H-\operatorname{dim} \phi(H)} 1_{\phi(F)}, \\
& \bar{\phi}_{*}^{\chi}\left(1_{F}\right)=(-1)^{\operatorname{dim} \phi(F)+\operatorname{dim} H-\operatorname{dim} \phi(H)} 1_{\text {ri }[\phi(F)]},
\end{aligned}
$$

where $H$ is a maximal affine subspace contained in $F$.

We summarize (11-20) as the following proposition.

Proposition 3.2. Let $\mathrm{V}$ and $\mathrm{W}$ be finite dimensional real vector spaces and $\phi$ an affine linear map from $\mathbf{V}$ to $\mathbf{W}$. Then $\phi^{*}$ is a linear map from $\mathbf{R}(\mathbf{W}, \mathcal{C} \cup \mathcal{O})$ to $\mathbf{R}(\mathbf{V}, \mathcal{C} \cup \mathcal{O})$, and $\phi_{*}^{\chi}, \bar{\phi}_{*}^{\chi}, \phi_{*}^{\bar{\chi}}, \bar{\phi}_{*}^{\bar{\chi}}$ are well-defined linear maps from $\mathbf{R}(\mathbf{V}, \mathcal{C} \cup \mathcal{O})$ to $\mathbf{R}(\mathbf{W}, \mathcal{C} \cup \mathcal{O})$. Moreover,

(1) $\phi^{*} \mathbf{R}(\mathbf{W}, \mathcal{O}) \subset \mathbf{R}(\mathbf{V}, \mathcal{O})$

(2) $\phi^{*} \mathbf{R}(\mathbf{W}, \mathcal{C}) \subset \mathbf{R}(\mathbf{V}, \mathcal{C})$

(3) $\phi_{*}^{\chi} \mathbf{R}(\mathbf{V}, \mathcal{O})=\mathbf{R}(\phi(\mathbf{V}), \mathcal{O})$

(4) $\phi_{*}^{\chi} \mathbf{R}(\mathbf{V}, \mathcal{C})=\mathbf{R}(\phi(\mathbf{V}), \mathcal{C})$

(5) $\bar{\phi}_{*}^{\chi} \mathbf{R}(\mathbf{V}, \mathcal{O})=\mathbf{R}(\phi(\mathbf{V}), \mathcal{C})$

(6) $\bar{\phi}_{*}^{\chi} \mathbf{R}(\mathbf{V}, \mathcal{C})=\mathbf{R}(\phi(\mathbf{V}), \mathcal{O})$,

(7) $\left.\phi_{*}^{\bar{\chi}} \mathbf{R}(\mathbf{V}), \mathcal{O}\right)=\mathbf{R}(\phi(\mathbf{V}), \mathcal{O})$,

(8) $\phi_{*}^{\bar{\chi}} \mathbf{R}(\mathbf{V}, \mathcal{C})=\mathbf{R}(\phi(\mathbf{V}), \mathcal{C})$

(9) $\bar{\phi}_{*}^{\bar{\chi}} \mathbf{R}(\mathbf{V}, \mathcal{O})=\mathbf{R}(\phi(\mathbf{V}), \mathcal{C})$

(10) $\bar{\phi}_{*}^{\bar{\chi}} \mathbf{R}(\mathbf{V}, \mathcal{C})=\mathbf{R}(\phi(\mathbf{V}), \mathcal{O})$.

Let $U$ be a relatively open convex set and $F$ a closed convex set of $\mathbf{V}$. It follows from (11-14) that

$$
\begin{aligned}
& \chi\left(\phi_{*}^{\chi}\left(1_{U}\right)\right)=(-1)^{\operatorname{dim} U-\operatorname{dim} \phi(U)} \chi(\phi(U))=\chi(U), \\
& \chi\left(\bar{\phi}_{*}^{\bar{\chi}}\left(1_{F}\right)\right)=(-1)^{\operatorname{dim} \phi(F)} \chi(\operatorname{ri}[\phi(F)])=\bar{\chi}(F), \\
& \bar{\chi}\left(\bar{\phi}_{*}^{\chi}\left(1_{U}\right)\right)=(-1)^{\operatorname{dim} U} \bar{\chi}(\operatorname{cl}[\phi(U)])=\chi(U), \\
& \bar{\chi}\left(\phi_{*}^{\bar{\chi}}\left(1_{F}\right)\right)=\bar{\chi}(\overline{\phi(F)})=\bar{\chi}(F) .
\end{aligned}
$$

Now, if the fiber $U \cap \phi^{-1}(w)$ for $w \in \phi(U)$ is a type II set, then $U$ has to be a type II set and thereby $\bar{\chi}\left(\phi_{*}^{\bar{\chi}}\left(1_{U}\right)\right)=\chi\left(\bar{\phi}_{*}^{\bar{\chi}}\left(1_{U}\right)\right)=\bar{\chi}(U)=0$ from (6); if, on the other hand, the fiber $U \cap \phi^{-1}(w)$ for $w \in \phi(U)$ is a type I set, then by (16) and (17),

$$
\begin{aligned}
& \bar{\chi}\left(\phi_{*}^{\bar{\chi}}\left(1_{U}\right)\right)=(-1)^{\operatorname{dim} U-\operatorname{dim} \phi(U)+\operatorname{dim} H-\operatorname{dim} \phi(H)} \bar{\chi}(\phi(U))=\bar{\chi}(U), \\
& \chi\left(\bar{\phi}_{*}^{\bar{\chi}}\left(1_{U}\right)\right)=(-1)^{\operatorname{dim} U-\operatorname{dim} H-\operatorname{dim} \phi(H)} \chi(\operatorname{cl}[\phi(U)])=\bar{\chi}(U) .
\end{aligned}
$$

Similarly, if the fiber $F \cap \phi^{-1}(w)$ for $w \in \phi(F)$ is a type II set, then $F$ should be a type II set. It follows from (4) that $\chi\left(\phi_{*}^{\chi}\left(1_{F}\right)\right)=\bar{\chi}\left(\bar{\phi}_{*}^{\chi}\left(1_{F}\right)\right)=\chi(F)=0$; while if the fiber $F \cap \phi^{-1}(w)$ for $w \in \phi(F)$ is a type I set, then by (19) and (20),

$$
\begin{aligned}
& \chi\left(\phi_{*}^{\chi}\left(1_{F}\right)\right)=(-1)^{\operatorname{dim} H-\operatorname{dim} \phi(U)} \chi(\phi(F))=\chi(F), \\
& \bar{\chi}\left(\bar{\phi}_{*}^{\chi}\left(1_{F}\right)\right)=(-1)^{\operatorname{dim} \phi(F)+\operatorname{dim} H-\operatorname{dim} \phi(F)} \bar{\chi}(\operatorname{ri}[\phi(F)])=\chi(F) .
\end{aligned}
$$


We conclude the above computation as the following proposition.

Proposition 3.3. Let $\mathbf{V}$ and $\mathbf{W}$ be finite dimensional real vector spaces and let $\phi$ be an affine linear map from $\mathbf{V}$ to $\mathbf{W}$. Then for any $f$ of $\mathbf{R}(\mathbf{V}, \mathcal{C} \cup \mathcal{O})$,

$$
\begin{aligned}
& \chi\left(\phi_{*}^{\chi} f\right)=\chi(f), \\
& \chi\left(\bar{\phi}_{*}^{\bar{\chi}} f\right)=\bar{\chi}(f), \\
& \bar{\chi}\left(\bar{\phi}_{*}^{\chi} f\right)=\chi(f), \\
& \bar{\chi}\left(\phi_{*}^{\bar{\chi}} f\right)=\bar{\chi}(f) .
\end{aligned}
$$

Next we consider the tensor product of the induced push-forward maps.

Proposition 3.4. Let $\phi, \psi: \mathbf{V}_{i} \longrightarrow \mathbf{W}_{i}$ be affine linear maps between finite dimensional real vector spaces, $i=1,2$. Then

$$
\begin{aligned}
& (\phi \otimes \psi)_{*}^{\chi}=\phi_{*}^{\chi} \otimes \psi_{*}^{\chi}, \\
& (\phi \otimes \psi)_{*}^{\bar{\chi}}=\phi_{*}^{\bar{\chi}} \otimes \psi_{*}^{\bar{\chi}}, \\
& (\overline{\phi \otimes \psi})_{*}^{\chi}=\bar{\phi}_{*}^{\chi} \otimes \bar{\psi}_{: k}^{\chi}, \\
& (\overline{\phi \otimes \psi})_{*}^{\bar{\chi}}=\bar{\phi}_{*}^{\bar{\chi}} \otimes \bar{\psi}_{: k}^{\bar{\chi}}
\end{aligned}
$$

on the tensor product $\mathbf{R}\left(\mathbf{V}_{1}, \mathcal{C} \cup \mathcal{O}\right) \otimes \mathbf{R}\left(\mathbf{V}_{2}, \mathcal{C} \cup \mathcal{O}\right)=\mathbf{R}\left(\mathbf{V}_{1} \times \mathbf{V}_{2}, \mathcal{C} \cup \mathcal{O}\right)$, where the map $\phi \otimes \psi$ is defined by $\phi \otimes \psi\left(v_{1}, v_{2}\right)=\left(\phi\left(v_{1}\right), \psi\left(v_{2}\right)\right),\left(v_{1}, v_{2}\right) \in \mathbf{V}_{1} \times \mathbf{V}_{2}$.

Proof. Let $A_{i} \subset \mathrm{V}_{i}$ be either simultaneously closed convex sets or simultaneously relatively open convex sets. Let $B_{i}\left(w_{i}, \delta\right)$ denote the open balls of radius $\delta$ centered at $w_{i}$ in $\mathbf{W}_{i}, i=1,2$. Then

$$
\begin{aligned}
& (\phi \otimes \psi)_{*}^{\chi}\left(1_{A_{1}} \otimes 1_{A_{2}}\right)\left(w_{1}, w_{2}\right)=(\phi \otimes \psi)_{*}^{\chi}\left(1_{A_{1} \times A_{2}}\right)\left(w_{1}, w_{2}\right) \\
& =\lim _{\delta \rightarrow 0} \chi\left[\left(A_{1} \times A_{2}\right) \cap(\phi \otimes \psi)^{-1}\left(\operatorname{cl} B\left(w_{1}, \delta\right) \times \operatorname{cl} B\left(w_{2}, \delta\right)\right)\right] \\
& =\lim _{\delta \rightarrow 0} \chi\left[\left(A_{1} \cap \phi^{-1}\left(\operatorname{cl} B\left(w_{1}, \delta\right)\right)\right) \times\left(A_{2} \cap \psi^{-1}\left(\operatorname{cl} B\left(w_{2}, \delta\right)\right)\right)\right] \\
& =\lim _{\delta \rightarrow 0} \chi\left[A_{1} \cap \phi^{-1}\left(\operatorname{cl} B\left(w_{1}, \delta\right)\right)\right] \lim _{\delta \rightarrow 0} \chi\left[A_{2} \cap \psi^{-1}\left(\operatorname{cl} B\left(w_{2}, \delta\right)\right)\right] \\
& =\phi_{*}^{\chi}\left(1_{A_{1}}\right)\left(w_{1}\right) \psi_{*}^{\chi}\left(1_{A_{2}}\right)\left(w_{2}\right) \\
& =\phi_{*}^{\chi}\left(1_{A_{1}}\right) \otimes \psi_{*}^{\chi}\left(1_{A_{2}}\right)\left(w_{1}, w_{2}\right) \\
& =\left(\phi_{*}^{\chi} \otimes \psi_{*}^{\chi}\right)\left(1_{A_{1}} \otimes 1_{A_{2}}\right)\left(w_{1}, w_{2}\right) ; \\
& (\phi \otimes \psi)_{*}^{\bar{\chi}}\left(1_{A_{1}} \otimes 1_{A_{2}}\right)\left(w_{1}, w_{2}\right)=(\phi \otimes \psi)_{*}^{\bar{\chi}}\left(1_{A_{1} \otimes A_{2}}\right)\left(w_{1}, w_{2}\right) \\
& =\lim _{\delta \rightarrow 0} \bar{\chi}\left[\left(A_{1} \times A_{2}\right) \cap(\phi \otimes \psi)^{-1}\left(\operatorname{cl} B\left(w_{1}, \delta\right) \times \operatorname{cl} B\left(w_{2}, \delta\right)\right)\right] \\
& =\lim _{\delta \rightarrow 0} \bar{\chi}\left[\left(A_{1} \cap \phi^{-1}\left(\operatorname{cl} B\left(w_{1}, \delta\right)\right)\right) \times\left(A_{2} \cap \psi^{-1}\left(\operatorname{cl} B\left(w_{2}, \delta\right)\right)\right)\right] \\
& =\lim _{\delta \rightarrow 0} \bar{\chi}\left[A_{1} \cap \phi^{-1}\left(\operatorname{cl} B\left(w_{1}, \delta\right)\right)\right] \lim _{\delta \rightarrow 0} \bar{\chi}\left[A_{2} \cap \psi^{-1}\left(\operatorname{cl} B\left(w_{2}, \delta\right)\right)\right] \\
& =\phi_{*}^{\bar{\chi}}\left(1_{A_{1}}\right)\left(w_{1}\right) \psi_{*}^{\bar{\chi}}\left(1_{A_{2}}\right)\left(w_{2}\right) \\
& =\phi_{*}^{\bar{\chi}}\left(1_{A_{1}}\right) \otimes \psi_{*}^{\bar{\chi}}\left(1_{A_{2}}\right)\left(w_{1}, w_{2}\right) \\
& =\left(\phi_{*}^{\bar{\chi}} \otimes \psi_{*}^{\bar{\chi}}\right)\left(1_{A_{1}} \otimes 1_{A_{2}}\right)\left(w_{1}, w_{2}\right) ;
\end{aligned}
$$




$$
\begin{aligned}
& (\overline{\phi \otimes \psi})_{*}^{\chi}\left(1_{A_{1}} \otimes 1_{A_{2}}\right)\left(w_{1}, w_{2}\right)=(\overline{\phi \otimes \psi})_{*}^{\chi}\left(1_{A_{1} \otimes A_{2}}\right)\left(w_{1}, w_{2}\right) \\
& =\lim _{\delta \rightarrow 0} \chi\left[\left(A_{1} \times A_{2}\right) \cap(\phi \otimes \psi)^{-1}\left(B\left(w_{1}, \delta\right) \times B\left(w_{2}, \delta\right)\right)\right] \\
& =\lim _{\delta \rightarrow 0} \chi\left[\left(A_{1} \cap \phi^{-1}\left(B\left(w_{1}, \delta\right)\right)\right) \times\left(A_{2} \cap \psi^{-1}\left(B\left(w_{2}, \delta\right)\right)\right)\right] \\
& =\lim _{\delta \rightarrow 0} \chi\left[A_{1} \cap \phi^{-1}\left(B\left(w_{1}, \delta\right)\right)\right] \lim _{\delta \rightarrow 0} \chi\left[A_{2} \cap \psi^{-1}\left(B\left(w_{2}, \delta\right)\right)\right] \\
& =\bar{\phi}_{*}^{\chi}\left(1_{A_{1}}\right)\left(w_{1}\right) \bar{\psi}_{*}^{\chi}\left(1_{A_{2}}\right)\left(w_{2}\right) \\
& =\left(\bar{\phi}_{*}^{\chi} \otimes \bar{\psi}_{*}^{\chi}\right)\left(1_{A_{1}} \otimes 1_{A_{2}}\right)\left(w_{1}, w_{2}\right) ; \\
& (\overline{\phi \otimes \psi})_{*}^{\bar{\chi}}\left(1_{A_{1}} \otimes 1_{A_{2}}\right)\left(w_{1}, w_{2}\right)=(\overline{\phi \otimes \psi})_{*}^{\bar{\chi}}\left(1_{A_{1} \otimes A_{2}}\right)\left(w_{1}, w_{2}\right) \\
& =\lim _{\delta \rightarrow 0} \bar{\chi}\left[\left(A_{1} \times A_{2}\right) \cap(\phi \otimes \psi)^{-1}\left(B\left(w_{1}, \delta\right) \times B\left(w_{2}, \delta\right)\right)\right] \\
& =\lim _{\delta \rightarrow 0} \bar{\chi}\left[\left(A_{1} \cap \phi^{-1}\left(B\left(w_{1}, \delta\right)\right)\right) \times\left(A_{2} \cap \psi^{-1}\left(B\left(w_{2}, \delta\right)\right)\right)\right] \\
& =\lim _{\delta \rightarrow 0} \bar{\chi}\left[A_{1} \cap \phi^{-1}\left(B\left(w_{1}, \delta\right)\right)\right] \lim _{\delta \rightarrow 0} \bar{\chi}\left[A_{2} \cap \psi^{-1}\left(B\left(w_{2}, \delta\right)\right)\right] \\
& =\bar{\phi}_{*}^{\bar{\chi}}\left(1_{A_{1}}\right)\left(w_{1}\right) \bar{\psi}_{*}^{\bar{\chi}}\left(1_{A_{2}}\right)\left(w_{2}\right) \\
& =\left(\bar{\phi}_{*}^{\bar{\chi}} \otimes \bar{\psi}_{*}^{\bar{\chi}}\right)\left(1_{A_{1}} \otimes 1_{A_{2}}\right)\left(w_{1}, w_{2}\right) .
\end{aligned}
$$

LEMMA 3.5. Let $\phi: \mathrm{U} \longrightarrow \mathrm{V}$ and $\psi: \mathrm{V} \longrightarrow \mathrm{W}$ be affine linear maps of finite dimensional real vector spaces. Let $E$ be either a closed convex set or a relatively open convex set of $\mathbf{U}$. Then for any fixed $w \in \psi \phi(E),(\psi \phi)^{-1}(w) \cap E$ is a type II set if and only if either $\phi^{-1}(v) \cap E$ is a type II set for all $v \in \psi^{-1}(w) \cap \phi(E)$ or $\psi^{-1}(w) \cap \phi(E)$ is a type II set.

Proof. It is clear that $\phi^{-1}(v) \cap E$ is non-empty for all $v \in \psi^{-1}(w) \cap \phi(E)$. Then

$$
(\psi \phi)^{-1}(w) \cap E=\bigcup_{v \in \psi^{-1}(w) \cap \phi(E)} \phi^{-1}(v) \cap E .
$$

This shows that the necessary and sufficient condition for $(\psi \phi)^{-1}(w) \cap E$ to be a type II set is that either $\phi^{-1}(v) \cap E$ is a type II set for all $v \in \psi^{-1}(w) \cap \phi(E)$ or $\psi^{-1}(w) \cap \phi(E)$ is a type II set.

Proposition 3.6. Let $\phi: \mathrm{U} \longrightarrow \mathrm{V}$ and $\psi: \mathrm{V} \longrightarrow \mathrm{W}$ be affine linear maps of finite dimensional real vector spaces. Then

(1) $(\psi \phi)_{*}^{\chi}=\psi_{*}^{\chi} \phi_{*}^{\chi}=\bar{\psi}_{*}^{\bar{\chi}} \bar{\phi}_{*}^{\chi}$,

(2) $(\psi \phi)_{*}^{\bar{\chi}}=\psi_{*}^{\bar{\chi}} \phi_{*}^{\bar{\chi}}=\bar{\psi}_{*}^{\chi} \bar{\phi}_{*}^{\bar{\chi}}$,

(3) $(\overline{\psi \phi})_{*}^{\chi}=\bar{\psi}_{*}^{\chi} \phi_{*}^{\chi}=\psi_{*}^{\bar{\chi}} \bar{\phi}_{*}^{\chi}$,

(4) $(\overline{\psi \phi})_{*}^{\bar{\chi}}=\bar{\psi}_{*}^{\bar{\chi}} \phi_{*}^{\bar{\chi}}=\psi_{*}^{\chi} \bar{\phi}_{*}^{\bar{\chi}}$,

(5) $\psi_{*}^{\bar{\chi}} \phi_{*}^{\chi}=\bar{\psi}_{*}^{\chi} \bar{\phi}_{*}^{\chi}$,

(6) $\psi_{*}^{\chi} \phi_{*}^{\bar{\chi}}=\bar{\psi}_{*}^{\bar{\chi}} \bar{\phi}_{*}^{\bar{\chi}}$,

(7) $\psi_{*}^{\chi} \bar{\phi}_{*}^{\chi}=\bar{\psi}_{*}^{\bar{\chi}} \phi_{*}^{\chi}$,

(8) $\psi_{*}^{\bar{\chi}} \bar{\phi}_{*}^{\bar{\chi}}=\bar{\psi}_{*}^{\chi} \phi_{*}^{\bar{\chi}}$.

Proof. Let $U$ be a relatively open convex set and $F$ a closed convex set of $\mathbf{U}$. It follows from (11-20) that

$$
\begin{aligned}
& (\psi \phi)_{*}^{\chi}\left(1_{U}\right)=\psi_{*}^{\chi} \phi_{*}^{\chi}\left(1_{U}\right)=\bar{\psi}_{*}^{\bar{\chi}} \bar{\phi}_{*}^{\chi}\left(1_{U}\right)=(-1)^{\operatorname{dim} U-\operatorname{dim} \psi \phi(U)} 1_{\psi \phi(U)}, \\
& (\psi \phi)_{*}^{\chi}\left(1_{F}\right)=\psi_{*}^{\bar{\chi}} \phi_{*}^{\bar{\chi}}\left(1_{F}\right)=\bar{\psi}_{*}^{\chi} \bar{\phi}_{*}^{\bar{\chi}}\left(1_{F}\right)=1_{\mathrm{cl}[\psi \phi(F)]}, \\
& (\overline{\psi \phi})_{*}^{\chi}\left(1_{U}\right)=\bar{\psi}_{*}^{\chi} \phi_{*}^{\chi}\left(1_{U}\right)=\psi_{*}^{\bar{\chi}} \bar{\phi}_{*}^{\chi}\left(1_{U}\right)=(-1)^{\operatorname{dim} U} 1_{\mathrm{cl}[\psi \phi(U)]}, \\
& (\overline{\psi \phi})_{*}^{\bar{\chi}}\left(1_{F}\right)=\bar{\psi}_{*}^{\bar{\chi}} \phi_{*}^{\bar{\chi}}\left(1_{F}\right)=\psi_{*}^{\chi} \bar{\phi}_{*}^{\bar{\chi}}\left(1_{F}\right)=(-1)^{\operatorname{dim} \psi \phi(F)} 1_{\operatorname{ri}[\psi \phi(F)]} .
\end{aligned}
$$


Now we divide the other situations into the following cases:

(Ia) The fiber $F \cap(\psi \phi)^{-1}(w)$ is a type I set for all $w \in \psi \phi(F)$. Then it follows from (19), (20), and (17) that

$$
\begin{aligned}
& (\psi \phi)_{*}^{\chi}\left(1_{F}\right)=\psi_{*}^{\chi} \phi_{*}^{\chi}\left(1_{F}\right)=\bar{\psi}_{*}^{\bar{\chi}} \bar{\phi}_{*}^{\chi}\left(1_{F}\right)=(-1)^{\operatorname{dim} H-\operatorname{dim} \psi \phi(H)} 1_{\mathrm{cl}[\psi \phi(F)]}, \\
& (\overline{\psi \phi})_{*}^{\chi}\left(1_{F}\right)=\bar{\psi}_{*}^{\chi} \phi_{*}^{\chi}\left(1_{F}\right)=\psi_{*}^{\bar{\chi}} \bar{\phi}_{*}^{\chi}\left(1_{F}\right)=(-1)^{\operatorname{dim} \psi \phi(F)+\operatorname{dim} H-\operatorname{dim} \psi \phi(H)} 1_{\mathrm{ri}[\psi \phi(F)]},
\end{aligned}
$$

where $H$ is a maximal affine subspace contained in $F$.

(Ib) The fiber $F \cap(\psi \phi)^{-1}(w)$ is a type II set for all $w \in \psi \phi(F)$. Then it follows from (18) and Lemma 3.5 that

$$
\begin{aligned}
& (\psi \phi)_{*}^{\chi}\left(1_{F}\right)=\psi_{*}^{\chi} \phi_{*}^{\chi}\left(1_{F}\right)=\bar{\psi}_{*}^{\bar{\chi}} \bar{\phi}_{*}^{\chi}\left(1_{F}\right)=0, \\
& (\overline{\psi \phi})_{*}^{\chi}\left(1_{F}\right)=\bar{\psi}_{*}^{\chi} \phi_{*}^{\chi}\left(1_{F}\right)=\psi_{*}^{\bar{\chi}} \bar{\phi}_{*}^{\chi}\left(1_{F}\right)=0 .
\end{aligned}
$$

(IIa) The fiber $U \cap(\psi \phi)^{-1}(w)$ is a type I set for all $w \in \psi \phi(U)$. Then by (16), (17), (19) and (20),

$$
\begin{aligned}
(\psi \phi)_{*}^{\bar{\chi}}\left(1_{U}\right) & =\psi_{*}^{\bar{x}} \phi_{*}^{\bar{\chi}}\left(1_{U}\right)=\bar{\psi}_{*}^{\chi} \bar{\phi}_{*}^{\bar{\chi}}\left(1_{U}\right) \\
& =(-1)^{\operatorname{dim} U-\operatorname{dim} \psi \phi(U)+\operatorname{dim} H-\operatorname{dim} \psi \phi(H)} 1_{\psi \phi(U)}, \\
(\overline{\psi \phi})_{*}^{\bar{\chi}}\left(1_{U}\right) & =\bar{\psi}_{*}^{\bar{\chi}} \phi_{*}^{\bar{\chi}}\left(1_{U}\right)=\psi_{*}^{\chi} \bar{\phi}_{*}^{\bar{\chi}}\left(1_{U}\right) \\
& =(-1)^{\operatorname{dim} U+\operatorname{dim} H-\operatorname{dim} \psi \phi(H)} 1_{c l}[\psi \phi(U)]
\end{aligned}
$$

where $H$ is a maximal affine subspace of $U$.

(IIb) The fiber $U \cap(\psi \phi)^{-1}(w)$ is a type II set for all $w \in \psi \phi(U)$. Then by (15) and Lemma 3.5,

$$
\begin{aligned}
& (\psi \phi)_{*}^{\bar{\chi}}\left(1_{U}\right)=\psi_{*}^{\bar{\chi}} \phi_{*}^{\bar{\chi}}\left(1_{U}\right)=\bar{\psi}_{*}^{\chi} \bar{\phi}_{*}^{\bar{\chi}}\left(1_{U}\right)=0, \\
& (\bar{\psi} \bar{\phi})_{*}^{\bar{\chi}}\left(1_{U}\right)=\bar{\psi}_{*}^{\bar{\chi}} \phi_{*}^{\bar{\chi}}\left(1_{U}\right)=\psi_{*}^{\chi} \bar{\phi}_{*}^{\bar{\chi}}\left(1_{U}\right)=0 .
\end{aligned}
$$

(IIIa) The fiber $\phi(U) \cap \psi^{-1}(w)$ is a type I set for all $w \in \psi \phi(U)$. Then by (11), (12), (16), (17), (19) and (20)

$$
\begin{aligned}
& \psi_{*}^{\bar{\chi}} \phi_{*}^{\chi}\left(1_{U}\right)=\bar{\psi}_{*}^{\chi} \bar{\phi}_{* *}^{\chi}\left(1_{U}\right)=(-1)^{\operatorname{dim} U-\operatorname{dim} \psi \phi(U)+\operatorname{dim} \phi(H)-\operatorname{dim} \psi \phi(H)} 1_{\psi \phi(U)}, \\
& \psi_{*}^{\chi} \bar{\phi}_{*}^{\chi}\left(1_{U}\right)=\bar{\psi}_{*}^{\bar{\chi}} \phi_{*}^{\chi}\left(1_{U}\right)=(-1)^{\operatorname{dim} U+\operatorname{dim} \phi(H)-\operatorname{dim} \psi \phi(H)} 1_{c l}[\psi \phi(U)]
\end{aligned}
$$

(IIIb) The fiber $\phi(U) \cap \psi^{-1}(w)$ is a type II set for all $w \in \psi \phi(U)$. Then by (11), (12), (15) and (18),

$$
\begin{aligned}
& \psi_{*}^{\bar{\chi}} \phi_{*}^{\chi}\left(1_{U}\right)=\bar{\psi}_{*}^{\chi} \bar{\phi}_{*}^{\chi}\left(1_{U}\right)=0, \\
& \psi_{*}^{\chi} \bar{\phi}_{*}^{\chi}\left(1_{U}\right)=\bar{\psi}_{*}^{\bar{\chi}} \phi_{*}^{\chi}\left(1_{U}\right)=0 .
\end{aligned}
$$

(IVa) The fiber $U \cap \phi^{-1}(w)$ is a type I set for all $v \in \phi(U)$. By (11-14), (16) and (17), we have

$$
\begin{aligned}
& \psi_{*}^{\chi} \phi_{*}^{\bar{\chi}}\left(1_{U}\right)=\bar{\psi}_{*}^{\bar{\chi}} \bar{\phi}_{*}^{\bar{\chi}}\left(1_{U}\right)=(-1)^{\operatorname{dim} U-\psi \phi(U)+\operatorname{dim} H-\phi(H)} 1_{\psi \phi(U)}, \\
& \psi_{*}^{\bar{\chi}} \bar{\phi}_{*}^{\bar{\chi}}\left(1_{U}\right)=\bar{\psi}_{*}^{\chi} \phi_{*}^{\bar{\chi}}\left(1_{U}\right)=(-1)^{\operatorname{dim} U+\operatorname{dim} H-\operatorname{dim} \phi(H)} 1_{c l}[\psi \phi(U)]^{\circ}
\end{aligned}
$$


(IVb) The fiber $U \cap \phi^{-1}(w)$ is a type II set for all $v \in \phi(U)$. By (15) we have

$$
\begin{aligned}
& \psi_{*}^{\chi} \phi_{*}^{\bar{\chi}}\left(1_{U}\right)=\bar{\psi}_{*}^{\bar{\chi}} \bar{\phi}_{*}^{\bar{\chi}}\left(1_{U}\right)=0, \\
& \psi_{*}^{\bar{\chi}} \bar{\phi}_{*}^{\bar{\chi}}\left(1_{U}\right)=\bar{\psi}_{*}^{\chi} \phi_{*}^{\bar{\chi}}\left(1_{U}\right)=0 .
\end{aligned}
$$

(Va) The fiber $F \cap \phi^{-1}(w)$ is a type I set for all $w \in \phi(F)$. It follows from (11-14), (19) and (20) that

$$
\begin{aligned}
& \psi_{*}^{\bar{\chi}} \phi_{*}^{\chi}\left(1_{F}\right)=\bar{\psi}_{*}^{\chi} \bar{\phi}_{*}^{\chi}\left(1_{F}\right)=(-1)^{\operatorname{dim} H-\operatorname{dim} \phi(H)} 1_{\mathrm{cl}[\psi \dot{\phi}(F)]} \\
& \psi_{*}^{\chi} \bar{\phi}_{*}^{\chi}\left(1_{F}\right)=\bar{\psi}_{*}^{\bar{\chi}} \phi_{*}^{\chi}\left(1_{F}\right)=(-1)^{\operatorname{dim} H-\operatorname{dim} \dot{\phi}(H)-\operatorname{dim} \psi \dot{\phi}(F)} 1_{\mathrm{ri}[\psi \phi(F)]} .
\end{aligned}
$$
that

(Vb) The fiber $F \cap \phi^{-1}(w)$ is a type II set for all $w \in \phi(F)$. It follows from (18)

$$
\begin{aligned}
& \psi_{*}^{\bar{\chi}} \phi_{*}^{\chi}\left(1_{F}\right)=\bar{\psi}_{*}^{\chi} \bar{\phi}_{*}^{\chi}\left(1_{F}\right)=0, \\
& \psi_{*}^{\chi} \bar{\phi}_{*}^{\chi}\left(1_{F}\right)=\bar{\psi}_{*}^{\bar{\chi}} \phi_{*}^{\chi}\left(1_{F}\right)=0 .
\end{aligned}
$$

(VIa) The fiber $\phi(F) \cap \psi^{-1}(w)$ is a type I set for all $w \in \psi \phi(F)$. Thus by (11-14), (16) and (17),

$$
\begin{aligned}
& \psi_{*}^{\bar{\chi}} \bar{\phi}_{*}^{\bar{\chi}}\left(1_{F}\right)=\bar{\psi}_{*}^{\chi} \phi_{*}^{\bar{\chi}}\left(1_{F}\right)=(-1)^{\operatorname{dim} \psi \phi(F)+\operatorname{dim} \phi(H)-\operatorname{dim} \psi \phi(H)} 1_{\mathrm{ri}[\psi \phi(F)]}, \\
& \psi_{*}^{\chi} \phi_{*}^{\bar{\chi}}\left(1_{F}\right)=\bar{\psi}_{*}^{\bar{\chi}} \bar{\phi}_{*}^{\bar{\chi}}\left(1_{F}\right)=(-1)^{\operatorname{dim} \phi(H)-\operatorname{dim} \psi \phi(H)} 1_{\psi \phi(F)} .
\end{aligned}
$$

(VIb) The fiber $\phi(F) \cap \psi^{-1}(w)$ is a type II set for all $w \in \psi \phi(F)$. Obviously, by (13-15) and (18)

$$
\begin{aligned}
& \psi_{*}^{\bar{\chi}} \bar{\phi}_{*}^{\bar{\chi}}\left(1_{F}\right)=\bar{\psi}_{*}^{\chi} \phi_{*}^{\bar{\chi}}\left(1_{F}\right)=0 \\
& \psi_{*}^{\chi} \phi_{*}^{\bar{\chi}}\left(1_{F}\right)=\bar{\psi}_{*}^{\bar{\chi}} \bar{\phi}_{*}^{\bar{\chi}}\left(1_{F}\right)=0
\end{aligned}
$$

4. Convolutions. Let $\mathbf{V}$ be a finite dimensional real vector space. Let $f$ and $g$ be simple functions both in $\mathbf{R}(\mathbf{V}, \mathcal{O})$ or both in $\mathbf{R}(\mathbf{V}, \mathcal{C})$. The convolutions $f * g$ and $f \bar{*} g$, with respect to the Euler measures $\chi$ and $\bar{\chi}$, are defined by

$$
\begin{aligned}
(f * g)(y) & =\int_{\mathbf{V}} f(x) g(y-x) \mathrm{d} \chi(x), \quad \forall y \in \mathbf{V}, \\
(f \bar{*} g)(y) & =\int_{\mathbf{V}} f(x) g(y-x) \mathrm{d} \bar{\chi}(x), \quad \forall y \in \mathbf{V} .
\end{aligned}
$$

It follows from the argument about $A$ and $B$ (see below) that for compact convex sets $K_{1}$ and $K_{2}$,

$$
1_{K_{1}} * 1_{K_{2}}=1_{K_{1}} * 1_{K_{2}}=1_{K_{1}+K_{2}} .
$$

This justifies why we call $*$ and $\bar{*}$ Minkowski multiplications with respect to the Euler measures $\chi$ and $\bar{\chi}$.

Let $A$ and $B$ be either both relatively open convex sets or both closed convex sets of $\mathbf{V}$. For any fixed $v \in \mathbf{V}$,

$$
1_{A}(x) 1_{B}(v-x)=1 \Leftrightarrow x \in A \cap(v-B) .
$$


Let $\alpha: \mathbf{V} \times \mathbf{V} \longrightarrow \mathbf{V}$ denote the vector addition, that is, $\alpha(x, y)=x+y$. Then the projection $\beta: \mathbf{V} \times \mathbf{V} \rightarrow \mathbf{V}$ onto its first component, that is, $\beta(x, y)=x$, is an affine one-to-one correspondence between $(A \times B) \cap \alpha^{-1}(v)$ and $A \cap(v-B)$ for each $v \in \mathbf{V}$. Therefore

$$
\begin{aligned}
&\left(1_{A} * 1_{B}\right)(v)=\chi(A \cap(v-B))=\chi\left((A \times B) \cap \alpha^{-1}(v)\right)=\alpha_{*}^{\chi}\left(1_{A \times B}\right)(v), \\
&\left(1_{A} \bar{*} 1_{B}\right)(v)=\bar{\chi}(A \cap(v-B))=\bar{\chi}\left((A \times B) \cap \alpha^{-1}(v)\right)=\alpha_{*}^{\bar{\chi}}\left(1_{A} \times 1_{B}\right)(v) .
\end{aligned}
$$

Thus for any $f, g \in \mathbf{R}(\mathbf{V}, \mathcal{O})$, or any $f, g \in \mathbf{R}(\mathbf{V}, \mathcal{C})$, the convolutions can be written as

$$
\begin{aligned}
f * g & =\alpha_{*}^{\chi}(f \otimes g), \\
f \bar{*} g & =\alpha_{*}^{\bar{\chi}}(f \otimes g),
\end{aligned}
$$

where $f \otimes g$ is the function on $\mathbf{V} \times \mathbf{V}$ defined by $(f \otimes g)(x, y)=f(x) g(y)$. This shows that $*$ and $\bar{*}$ are bilinear operations on both $\mathbf{R}(\mathbf{V}, \mathcal{O})$ and $\mathbf{R}(\mathbf{V}, \mathcal{C})$. The bilinearity of $*$ and $\bar{*}$ follows from the linearity of $\alpha_{*}^{\chi}$ and $\alpha_{*}^{\bar{\chi}}$ and the bilinearity of $\otimes$. Moreover, for relatively open convex sets $U_{1}, U_{2}$ and closed convex sets $F_{1}, F_{2}$, we have the explicit formulas:

$$
\begin{aligned}
1_{U_{1}} * 1_{U_{2}} & =(-1)^{\operatorname{dim} U_{1}+\operatorname{dim} U_{2}-\operatorname{dim}\left(U_{1}+U_{2}\right)} 1_{U_{1}+U_{2}}, \\
1_{F_{1}} * 1_{F_{2}} & =\chi\left(\operatorname{rec}\left(F_{1}\right) \cap \operatorname{rec}\left(-F_{2}\right)\right) 1_{c l}\left[F_{1}+F_{2}\right] \\
1_{U_{1}} 1_{U_{2}} & =(-1)^{\operatorname{dim} U_{1}+\operatorname{dim} U_{2}-\operatorname{dim}\left(U_{1}+U_{2}\right)} \chi\left(\operatorname{rec}\left(U_{1}\right) \cap \operatorname{rec}\left(-U_{2}\right)\right) 1_{U_{1}+U_{2}}, \\
1_{F_{1}} * 1_{F_{2}} & =1_{c l}{ }_{\left[F_{1}+F_{2}\right]} .
\end{aligned}
$$

Now if $F_{1} \cap\left(v-F_{2}\right)$ is a type II set for one $v \in F_{1}+F_{2}$, then $1_{F_{1}} * 1_{F_{2}}=0$. Otherwise, $F_{1} \cap\left(v-F_{2}\right)$ are type I sets for all $v \in F_{1}+F_{2}$. We then have

$$
1_{F_{1}} * 1_{F_{2}}=(-1)^{\operatorname{dim} H_{1}+\operatorname{dim} H_{2}-\operatorname{dim}\left(H_{1}+H_{2}\right)} 1_{\mathrm{cl}\left[F_{1}+F_{2}\right]},
$$

where $H_{1}$ is a maximal affine subspace contained in $F_{1}$ and $H_{2}$ a maximal affine subspace contained in $F_{2}$.

Similarly, if $U_{1} \cap\left(v-U_{2}\right)$ is a type II set, then $1_{U_{1}} \bar{*} 1_{U_{2}}=0$; while if $U_{1} \cap\left(v-U_{2}\right)$ is a type I set, then

$$
1_{U_{1}} \bar{*} 1_{U_{2}}=(-1)^{\operatorname{dim} U_{1}+\operatorname{dim} U_{2}-\operatorname{dim}\left(U_{1}+U_{2}\right)+\operatorname{dim} H_{1}+\operatorname{dim} H_{2}-\operatorname{dim}\left(H_{1}+H_{2}\right)} 1_{U_{1}+U_{2}},
$$

where $H_{1}$ is a maximal affine subspace contained in $U_{1}$ and $H_{2}$ a maximal affine subspace contained in $U_{2}$.

Let $A, B, C$ be convex sets of $\mathbf{V}$, either all closed or all relatively open. Then for any fixed $v \in \mathbf{V}$,

$$
(A \times B) \cap \alpha^{-1}(v-C)=\left\{(x, y) \mid 1_{A}(x) 1_{B}(y) 1_{C}(v-x-y)=1\right\} .
$$

Let $\gamma: \mathrm{V} \times \mathbf{V} \longrightarrow \mathbf{V} \times \mathbf{V}$ be the linear map defined by $\gamma(x, y)=(x, x+y)$. Then

$$
\gamma\left[(A \times B) \cap \alpha^{-1}(v-C)\right]=\left\{(x, y) \mid 1_{A}(x) 1_{B}(y-x) 1_{C}(v-y)=1\right\} .
$$

Thus by the definition of convolution $*$, it follows that for each $v \in \mathbf{V}$,

$$
1_{A} *\left(1_{B} * 1_{C}\right)(v)=\int 1_{A}(x)\left(1_{B} * 1_{C}\right)(v-x) d \chi(x)
$$




$$
\begin{aligned}
& =\iint 1_{A}(x) 1_{B}(y) 1_{C}(v-x-y) d \chi(y) d \chi(x) \\
& =\iint 1_{A}(x) 1_{B}(y) 1_{C}(v-x-y) d \chi(x, y) \\
& =\chi\left[(A \times B) \cap \alpha^{-1}(v-C)\right], \\
\left(1_{A} * 1_{B}\right) * 1_{C}(v) & =\int\left(1_{A} * 1_{B}\right)(y) 1_{C}(v-y) d \chi(y) \\
& =\iint 1_{A}(x) 1_{B}(y-x) 1_{C}(v-y) d \chi(x) d \chi(y) \\
& =\iint 1_{A}(x) 1_{B}(y-x) 1_{C}(v-y) d \chi(x, y) \\
& =\chi\left[\gamma\left((A \times B) \cap \alpha^{-1}(v-C)\right)\right] \\
& =\chi\left[(A \times B) \cap \alpha^{-1}(v-C)\right] .
\end{aligned}
$$

This means that

$$
\left(1_{A} * 1_{B}\right) * 1_{C}=1_{A} *\left(1_{B} * 1_{C}\right) .
$$

Therefore, for functions $f, g, h \in \mathbf{R}(\mathbf{V}, \mathcal{C})$ and for $f, g, h \in \mathbf{R}(\mathbf{V}, \mathcal{O})$, we have

$$
(f * g) * h=f *(g * h) .
$$

The associativity of the Minkowski multiplication $\bar{*}$ can be similarly obtained. It follows from Proposition 3.3 that

$$
\begin{aligned}
\chi(f * g) & =\chi\left[\alpha_{*}^{\chi}(f \otimes g)\right] \\
& =\chi(f \otimes g) \\
& =\chi(f) \chi(g), \\
\bar{\chi}(f \bar{*} g) & =\bar{\chi}\left[\alpha_{*}^{\bar{\chi}}(f \otimes g)\right] \\
& =\bar{\chi}(f \otimes g) \\
& =\bar{\chi}(f) \bar{\chi}(g) .
\end{aligned}
$$

We conclude the above observations as the following theorem.

THEOREM 4.1. For any finite dimensional real vector space $\mathbf{V}, \mathbf{R}(\mathbf{V}, \mathcal{O})$ and $\mathbf{R}(\mathbf{V}, \mathcal{C})$ are commutative algebras with identity $1_{\{\text {o\} }}$ under the multiplications $*$ and $\bar{*}$, $\chi$ is an algebra homomorphism from the Minkowski algebras $\mathbf{R}(\mathbf{V}, \mathcal{O}, *)$ and $\mathbf{R}(\mathbf{V}, \mathcal{C}, *)$ to $\mathbf{R}$, and $\bar{\chi}$ is an algebra homomorphism from $\mathbf{R}(\mathbf{V}, \mathcal{O}, \bar{*})$ and $\mathbf{R}(\mathbf{V}, \mathcal{C}, \bar{*})$ to $\mathbf{R}$.

Let $\phi$ be a linear map from a vector space $V$ to a vector space $\mathbf{W}$. Then the diagram

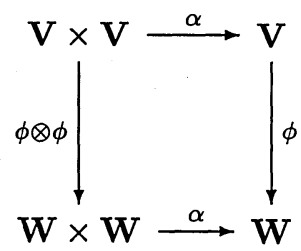


is commutative, where $(\phi \otimes \phi)(x, y)=(\phi(x), \phi(y))$. It follows from Proposition 3.6 that

$$
\begin{aligned}
& \phi_{*}^{\chi}(f * g)=\phi_{*}^{\chi} \alpha_{*}^{\chi}(f \otimes g) \\
& =(\phi \alpha)_{*}^{\chi}(f \otimes g) \\
& =(\alpha(\phi \otimes \phi))_{*}^{\chi}(f \otimes g) \\
& =\alpha_{*}^{\chi}(\phi \otimes \phi)_{*}^{\chi}(f \otimes g) \\
& =\alpha_{*}^{\chi}\left(\phi_{*}^{\chi} \otimes \phi_{*}^{\chi}\right)(f \otimes g) \\
& =\alpha_{*}^{\chi}\left(\phi_{*}^{\chi}(f) \otimes \phi_{*}^{\chi}(g)\right) \\
& =\phi_{*}^{\chi}(f) * \phi_{*}^{\chi}(g) \text {. } \\
& \phi_{*}^{\bar{\chi}}(f \bar{*} g)=\phi_{*}^{\bar{\chi}} \alpha_{*}^{\bar{\chi}}(f \otimes g) \\
& =(\phi \alpha)_{*}^{\bar{\chi}}(f \otimes g) \\
& =(\alpha(\phi \otimes \phi))_{*}^{\bar{\chi}}(f \otimes g) \\
& =\alpha_{*}^{\bar{\chi}}(\phi \otimes \phi)_{*}^{\bar{\chi}}(f \otimes g) \\
& =\alpha_{*}^{\bar{\chi}}\left(\phi_{*}^{\bar{\chi}} \otimes \phi_{*}^{\bar{\chi}}\right)(f \otimes g) \\
& =\alpha_{*}^{\bar{\chi}}\left(\phi_{*}^{\bar{\chi}}(f) \otimes \phi_{*}^{\bar{\chi}}(g)\right) \\
& =\phi_{*}^{\bar{\chi}}(f) \bar{*} \phi_{*}^{\bar{\chi}}(g) \text {. } \\
& \bar{\phi}_{*}^{\chi}(f * g)=\bar{\phi}_{*}^{\chi} \alpha_{*}^{\chi}(f \otimes g) \\
& =(\overline{\phi \alpha})_{*}^{\chi}(f \otimes g) \\
& =(\overline{\alpha(\phi \otimes \phi)})_{*}^{\chi}(f \otimes g) \\
& =\alpha_{*}^{\bar{\chi}}(\overline{\phi \otimes \phi})_{*}^{\chi}(f \otimes g) \\
& =\alpha_{*}^{\bar{\chi}}\left(\bar{\phi}_{*}^{\chi} \otimes \bar{\phi}_{*}^{\chi}\right)(f \otimes g) \\
& =\alpha_{*}^{\bar{\chi}}\left(\bar{\phi}_{*}^{\chi}(f) \otimes \bar{\phi}_{*}^{\chi}(g)\right) \\
& =\bar{\phi}_{*}^{\chi}(f) \bar{*} \bar{\phi}_{*}^{\chi}(g) \text {. } \\
& \bar{\phi}_{*}^{\bar{\chi}}(f \bar{*} g)=\bar{\phi}_{*}^{\bar{\chi}} \alpha_{*}^{\bar{\chi}}(f \otimes g) \\
& \begin{aligned}
= & (\overline{\phi \alpha})_{*}^{\bar{\chi}}(f \otimes g) \\
& (\overline{\alpha(\phi \otimes \phi)})_{*}^{\bar{\chi}}(f \otimes g)
\end{aligned} \\
& =\alpha_{*}^{\bar{\chi}}(\overline{\phi \otimes \phi})_{*}^{\bar{\chi}}(f \otimes g) \\
& =\alpha_{*}^{\bar{\chi}}\left(\phi_{*}^{\bar{\chi}} \otimes \phi_{*}^{\bar{\chi}}\right)(f \otimes g) \\
& =\alpha_{*}^{\bar{\chi}}\left(\bar{\phi}_{*}^{\bar{\chi}}(f) \otimes \bar{\phi}_{*}^{\bar{\chi}}(g)\right) \\
& =\bar{\phi}_{*}^{\bar{\chi}}(f) * \bar{\phi}_{*}^{\bar{\chi}}(g) \text {. }
\end{aligned}
$$

THEOREM 4.2. Let $\phi$ be a linear map of real vector spaces $\mathbf{V}$ to $\mathbf{W}$. Then $\phi_{*}^{\chi}$ is an algebra homomorphism from $\mathbf{R}(\mathbf{V}, \mathcal{O}, *)$ to $\mathbf{R}(\mathbf{W}, \mathcal{O}, *)$, and from $\mathbf{R}(\mathbf{V}, \mathcal{C}, *)$ to $\mathbf{R}(\mathbf{W}, \mathcal{C}, *) ; \phi_{*}^{\bar{\chi}}$ is an algebra homomorphism from $\mathbf{R}(\mathbf{V}, \mathcal{O}, \bar{*})$ to $\mathbf{R}(\mathbf{W}, \mathcal{O}, \bar{*})$, and from $\mathbf{R}(\mathbf{V}, \mathcal{C}, \bar{*})$ to $\mathbf{R}(\mathbf{W}, \mathcal{C}, \bar{*}) ; \bar{\phi}_{*}^{\chi}$ is an homomorphism from $\mathbf{R}(\mathbf{V}, \mathcal{O}, *)$ to $\mathbf{R}(\mathbf{W}, \mathcal{C}, \bar{*})$, and from $\mathbf{R}(\mathbf{V}, \mathcal{C}, *)$ to $\mathbf{R}(\mathbf{W}, \mathcal{O}, \bar{*})$; and $\bar{\phi}_{*}^{\bar{\chi}}$ is an algebra homomorphism from $\mathbf{R}(\mathbf{V}, \mathcal{O}, \bar{*})$ to $\mathbf{R}(\mathbf{W}, \mathcal{C}, *)$, and from $\mathbf{R}(\mathbf{V}, \mathcal{C}, \bar{*})$ to $\mathbf{R}(\mathbf{W}, \mathcal{O}, *)$. 
Let $i d$ denote the identity map from $\mathbf{V}$ to itself. Obviously, $i d_{*}^{\chi}=i d_{*}^{\bar{\chi}}$ and $\overline{i d}_{*}^{\chi}=\overline{i d_{*}^{\bar{\chi}}}$. So we denote $i d_{*}^{\chi}$ and $i d_{*}^{\bar{\chi}}$ by $i d_{*}$ and $\overline{i d_{*}}$ respectively. Notice that $i d_{*}$ is the identity map, while $\overline{i d_{*}}$ is an automorphism. Moreover, it follows from (1), (2), (5), and (6) of Proposition 3.6 that

$$
\overline{i d}_{*}^{2}=\overline{i d}_{*} \overline{i d}_{*}=\text { identity }
$$

This means that $\overline{i d}_{*}$ is an involution. The algebra isomorphism $\overline{i d}_{*}$ from $\mathbf{R}(\mathbf{V}, \mathcal{O}, *)$ onto $\mathbf{R}(\mathbf{V}, \mathcal{C}, \bar{*})$ is called Sallee-Shephard mapping [13].

THEOREM 4.3. $\overline{i d}_{*}$ is a linear involution on $\mathbf{R}(\mathbf{V}, \mathcal{O} \cup \mathcal{C})$, and it is an algebra isomorphism from $\mathbf{R}(\mathbf{V}, \mathcal{O}, *)$ onto $\mathbf{R}(\mathbf{V}, \mathcal{C}, \bar{*})$, from $\mathbf{R}(\mathbf{V}, \mathcal{O}, \bar{*})$ onto $\mathbf{R}(\mathbf{V}, \mathcal{C}, *)$, from $\mathbf{R}(\mathbf{V}, \mathcal{C}, *)$ onto $\mathbf{R}(\mathbf{V}, \mathcal{O}, \bar{*})$, and from $\mathbf{R}(\mathbf{V}, \mathcal{C}, \bar{*})$ onto $\mathbf{R}(\mathbf{V}, \mathcal{O}, *)$.

Let $P$ be a relatively open convex polyhedron. Clearly, $1_{\mathrm{cl} P}=\sum_{F \leq P} 1_{F}$. Then we have the Möbius inversion

$$
(-1)^{\operatorname{dim} P} 1_{P}=\overline{i d}_{*}\left(1_{\mathrm{cl} P}\right)=\sum_{F \leq P} \overline{i d}_{*}\left(1_{F}\right)=\sum_{F \leq P}(-1)^{\operatorname{dim} F} 1_{\mathrm{cl} F} .
$$

The induced forward maps can also be defined for polyconvex maps, that is, the maps whose graphs are polyconvex sets [3].

5. Embedding. Let $\mathbf{V}$ be a finite dimensional real vector space. We denote by $\mathbf{R}[\mathbf{V}]$ the vector space generated by the elements of $\mathbf{V}$ over $\mathbf{R}$. Each element $f$ of $\mathbf{R}[\mathbf{V}]$ can be written as $\sum_{i} a_{i} 1_{\left\{p_{i}\right\}}$. The vector addition of $\mathbf{V}$ induces a multiplication $*$ on $\mathbf{R}[\mathbf{V}]$, given by

$$
\sum_{i} a_{i} 1_{\left\{p_{i}\right\}} * \sum_{j} b_{j} 1_{\left\{q_{j}\right\}}=\sum_{i, j} a_{i} b_{j} 1_{\left\{p_{i}+q_{j}\right\}} .
$$

Obviously, $\mathbf{R}[\mathbf{V}]$ is a subalgebra of $\mathbf{R}(\mathbf{V}, \mathcal{C} \cup \mathcal{O})$.

If $\mathbf{V}$ is the line $\mathbf{R}$ of real numbers, we have the boundary operators $\partial^{+}$and $\partial^{-}$on $\mathbf{R}(\overline{\mathbf{R}}, \mathcal{C} \cup \mathcal{O})$, defined by

$$
\begin{aligned}
& \partial^{+}(f)(x)=f(x)-f\left(x^{+}\right), \quad \forall x \in \overline{\mathbf{R}}, \\
& \partial^{-}(f)(x)=f(x)-f\left(x^{-}\right), \quad \forall x \in \overline{\mathbf{R}},
\end{aligned}
$$

where $f\left(-\infty^{+}\right)=\lim _{x \rightarrow-\infty} f(x), f\left(+\infty^{-}\right)=\lim _{x \rightarrow+\infty} f(x)$, and $f\left(-\infty^{-}\right)=f\left(+\infty^{+}\right)$ $=0$. For $a, b, c, d \in \overline{\mathbf{R}}$, if $a \leq b$ and $c \leq d$, then $\partial^{+} 1_{[a, b]}=1_{\{b\}}, \partial^{-} 1_{[a, b]}=1_{\{a\}}$, and $\partial^{+}\left(1_{[a, b]} * 1_{[c, d]}\right)=\partial^{+}\left(1_{[a, b]}\right) * \partial^{+}\left(1_{[c, d]}\right)$. Thus $\partial^{+}$and $\partial^{-}$are algebra homomorphisms. Here we should be aware of the fact that we only consider the Minkowski multiplications of functions $f$ and $g$ such that $f(-\infty) g(+\infty)=f(+\infty) g(-\infty)=0$.

We now introduce the summation operators $s^{+}$and $s^{-}$on $\mathbf{R}(\overline{\mathbf{R}}, \mathcal{C} \cup \mathcal{O})$, defined by

$$
\begin{aligned}
& s^{+}(f)(x)=\chi\left(f \cdot 1_{[x,+\infty]}\right), \quad \forall x \in \overline{\mathbf{R}}, \\
& s^{-}(f)(x)=\chi\left(f \cdot 1_{(x,+\infty]}\right), \quad \forall x \in \overline{\mathbf{R}} .
\end{aligned}
$$

Notice that $s^{+}\left(1_{\{b\}}\right)=1_{[-\infty, b]}$ and $s^{-}\left(1_{\{a\}}\right)=1_{[-\infty, a)}$. Then $1_{[a, b]}=s^{+}\left(1_{\{b\}}\right)-$ $s^{-}\left(1_{\{a\}}\right)$. Thus for any $f \in \mathbf{R}(\overline{\mathbf{R}}, \mathcal{C} \cup \mathcal{O})$, 


$$
f=\left(s^{+} \partial^{+}-s^{-} \partial^{-}\right) f^{*}
$$

Definition 5.1. For each convex chain $f \in \mathbf{R}(\mathbf{V}, \mathcal{C} \cup \mathcal{O})$, the support function of $f$ is the map $H_{f}: \mathbf{V}^{*} \longrightarrow \mathbf{R}[\overline{\mathbf{R}}]$, defined by $H_{f}(\ell)=\partial^{+} \ell_{*}^{\chi}(f), \ell \in \mathbf{V}^{*}$.

The support function $H_{f}$ of a convex chain $f$ can be viewed as a multivalued function on $\mathbf{V}^{*}$ with multiplicities of real numbers. If $f$ is the indicator function of a closed convex set $F$ or a relatively open convex set $U, H_{f}$ is alternatively denoted by $H_{F}$ and $H_{U}$ respectively. We denote the set of all functions from $\mathbf{V}^{*}$ to $\mathbf{R}[\overline{\mathbf{R}}]$ by $F\left(\mathbf{V}^{*}, \mathbf{R}[\overline{\mathbf{R}}]\right)$. Since $\mathbf{R}[\overline{\mathbf{R}}]$ is a commutative algebra with identity, $F\left(V^{*}, \mathbf{R}[\overline{\mathbf{R}}]\right)$ is also a commutative algebra with identity, and there is an algebra homomorphism $\chi: F\left(\mathbf{V}^{*}, \mathbf{R}[\overline{\mathbf{R}}]\right) \longrightarrow F\left(\mathbf{V}^{*}, \mathbf{R}\right)$, given by

$$
\chi(\alpha)(\ell)=\sum_{i} a_{i}(\ell)
$$

where $\alpha(\ell)=\sum_{i} a_{i}(\ell) 1_{\left\{p_{i}(\ell)\right\}}, \ell \in \mathbf{V}^{*}$.

Let $E$ be a convex set of $\mathbf{V}$. We denote by $\langle E\rangle$ the linear subspace that is the translation of the flat that $E$ spans, and denote by $E^{\perp}$ the orthogonal subspace of $\langle E\rangle$, that is,

$$
E^{\perp}=\left\{\ell \in \mathbf{V}^{*} \mid \ell(u)=0, \forall u \in\langle E\rangle\right\} .
$$

For a convex cone $C \subset \mathbf{V}$, its normal cone $C^{*}$ is defined by

$$
C^{*}=\left\{\ell \in \mathbf{V}^{*} \mid \ell(u) \leq 0, \forall u \in C\right\} .
$$

The following Lemma 5.2, Lemma 5.3, and Lemma 5.4 will be needed to prove the embedding Theorem 5.5.

LEMMA 5.2. Let $F$ be a closed convex set and $S$ a vector subspace of a finite dimensional real vector space $\mathbf{V}$. If $(x+S) \cap F$ is non-empty for a vector $x \in \mathbf{V}$, then $(x+S) \cap F$ and $S \cap \operatorname{rec} F$ are the same type of closed convex sets, and

$$
\begin{aligned}
& \chi[(x+S) \cap F]=\chi(S \cap \operatorname{rec} F), \\
& \bar{\chi}[(x+S) \cap F]=\bar{\chi}(S \cap \operatorname{rec} F) .
\end{aligned}
$$

Proof. It is clear from the definition of the recession cone that $L$ is a onedimensional subspace, or a ray emanating from the origin in $\operatorname{rec} F$, if and only if, $x+L$ is a line or half-line in $F$ for every $x \in F$. Then $(x+S) \cap F$ and $S \cap \operatorname{rec} F$ are the same type of closed convex sets. Moreover, the linearity parts of both sets are the same. Thus by (4) and (5) they have the same Euler characteristics.

LEMMA 5.3. Let $F$ be a closed convex set and $U$ a relatively open convex set.

(i) If $F=K \oplus W$ is a type I set in (4), then

$$
H_{F}(\ell)= \begin{cases}(-1)^{\operatorname{dim} W} 1_{\left\{h_{F}^{+}(\ell)\right\}} & \text { for } \ell \in W^{\perp} \\ (-1)^{\operatorname{dim} W} 1_{\{-\infty\}} & \text { for } \ell \notin W^{\perp}\end{cases}
$$


(ii) If $F=M \oplus W \oplus L$ is a type II set in (4), then

$$
H_{F}(\ell)= \begin{cases}(-1)^{\operatorname{dim} W}\left(1_{\left\{h_{F}^{+}(\ell)\right\}}-1_{\{-\infty\}}\right) & \text { for } \ell \in \operatorname{ri}(\operatorname{rec} F)^{*} \\ 0 & \text { for } \ell \notin \operatorname{ri~}(\operatorname{rec} F)^{*}\end{cases}
$$

$$
H_{U}(\ell)=(-1)^{\operatorname{dim} U} 1_{\left\{h_{\bar{U}}(\ell)\right\}} .
$$

Proof. Without loss of generality, we may assume that $\mathbf{V}$ is endowed with a positive definite inner product and $\mathbf{V}^{*}$ is identified to $\mathbf{V}$. So a linear functional $\ell$ on $\mathbf{V}$ is also a vector of $\mathbf{V}$.

(i) Notice that the subspace $W$ in the decomposition of $F$ must be a proper subspace of $\mathbf{V}$. For $\ell \in W^{\perp}$, evidently, $W \subset \ell^{\perp}$, and the orthogonal projection of $F$ onto the line $\mathbf{R} \ell$ is $\ell(K)$. Then $\ell_{*}^{\chi}\left(1_{F}\right)=(-1)^{\operatorname{dim} W_{1}} 1_{\ell(K)}$, where $\ell(K)$ is a closed segment of the form $\left[a_{\ell}, h_{F}^{+}(\ell)\right]$. For $\ell \notin W^{\perp}$, the intersection $W \cap \ell^{\perp}$ is a subspace of dimension $\operatorname{dim} W-1$, and the orthogonal projection of $F$ onto the line $\mathbf{R} \ell$ is just $\mathbf{R} \ell$. Then $\ell_{*}^{\chi}\left(1_{F}\right)=(-1)^{\operatorname{dim} W-1} 1_{\mathbf{R}}$. We write the two cases together as

$$
\ell_{*}^{\chi}\left(1_{F}\right)= \begin{cases}(-1)^{\operatorname{dim} W} 1_{\ell(K)} & \text { for } \ell \in W^{\perp} \\ (-1)^{\operatorname{dim} W-1} 1_{\mathbf{R}} & \text { for } \ell \notin W^{\perp}\end{cases}
$$

Apply the operator $\partial^{+}$to both sides, (i) follows immediately.

(ii) Note that for any closed convex cone $C, \ell \in \mathrm{ri} C^{*}$ if and only if $\ell^{\perp} \cap C$ is the linearity of $C$. Moreover, if the linearity of $C$ is not $C$ itself, then $\ell \notin \operatorname{ri} C^{*}$ if and only if $\ell^{\perp} \cap C$ is a type II set. Now let $C=\operatorname{rec} F$. The linearity of $\operatorname{rec} F$ is $W$.

For $\ell \in \operatorname{ri}(\operatorname{rec} F)^{*}$, if $\left(\ell^{\perp}+x\right) \cap F \neq \emptyset$ for some $x \in \mathbf{V}$, then by Lemma 5.2 , the intersection $\left(\ell^{\perp}+x\right) \cap F$ is a flat of dimension $\operatorname{dim} W$, and $\ell(F)$ is a half-open interval of the form $\left(-\infty, h_{F}^{+}(\ell)\right]$. So $\ell_{*}^{\chi}\left(1_{F}\right)=(-1)^{\operatorname{dim} W} 1_{\left(-\infty, h_{F}^{+}(\ell)\right]}$.

For $\ell \notin \mathrm{ri}(\operatorname{rec} F)^{*}$, if $\left(\ell^{\perp}+x\right) \cap F \neq \emptyset$ for some $x \in \mathbf{V}$, then by Lemma 5.2 , the intersection $\left(\ell^{\perp}+x\right) \cap F$ is a type II set. So $\ell_{*}^{\chi}\left(1_{F}\right)=0$. Thus we have

$$
\ell_{*}^{\chi}\left(1_{F}\right)= \begin{cases}(-1)^{\operatorname{dim} W} 1_{\left(-\infty, h_{F}^{+}(\ell)\right]} & \text { for } \ell \in \operatorname{ri}(\operatorname{rec} F)^{*} \\ 0 & \text { for } \ell \notin \operatorname{ri}(\operatorname{rec} F)^{*}\end{cases}
$$

Apply the operator $\partial^{+}$again, the formula (ii) follows.

(iii) For $\ell \in\langle U\rangle^{\perp}$, then $\ell(U)$ is a singleton. Obviously, $\partial^{+} \ell_{*}^{\chi}\left(1_{U}\right)=(-1)^{\operatorname{dim} U} 1_{h_{U}^{-}(\ell)}$. Now for $\ell \notin U^{\perp}, \ell(U)$ will be an open interval $\left(h_{U}^{-}(\ell), b\right)$, and $\left(x+\ell^{\perp}\right) \cap U$ has the dimension of $\operatorname{dim} U-1$ if the intersection is non-empty. Notice that for any open interval $(a, b), \partial^{+} 1_{(a, b)}=-1_{\{a\}}$. We thus have $\partial^{+} \ell_{*}^{\chi}\left(1_{U}\right)=(-1)^{\operatorname{dim} U} 1_{\left\{h_{U}^{-}(\ell)\right\}}$.

Using an idea of [15] and [18] for support functions of polytopes, we define the bilinear map $I: \mathbf{R}[\overline{\mathbf{R}}] \times \mathbf{R}[\overline{\mathbf{R}}] \longrightarrow \mathbf{R}$ by

$$
I\left(1_{\{a\}}, 1_{\{b\}}\right)= \begin{cases}1 & \text { for } a \leq b \\ 0 & \text { for } a>b\end{cases}
$$

through linearity. In fact, $I$ can be defined explicitly without extension by

$$
I(f, g)=\chi\left(f \cdot s^{+} g\right), \quad \forall f, g \in \mathbf{R}[\overline{\mathbf{R}}] .
$$

For each $f \in F\left(V^{*}, \mathbf{R}[\overline{\mathbf{R}}]\right)$ and $x \in \mathbf{V}$, we define the function $\phi(f, x, \cdot): \mathbf{V}^{*} \longrightarrow \mathbf{R}$ by

$$
\phi(f, x, \ell)=I\left(1_{\{\ell(x)\}}, f(\ell)\right) .
$$


LEMMA 5.4. Let $F$ be a closed convex set and $U$ a relatively open convex set.

(i) If $F$ has a direct sum decomposition $F=K \oplus W$ in (4), then

$$
\phi\left(H_{F}, x, \ell\right)= \begin{cases}(-1)^{\operatorname{dim} W} & \text { for } x \in F, \ell \in W^{\perp} \\ 0 & \text { for } x \in F, \ell \notin W^{\perp} \\ (-1)^{\operatorname{dim} W} & \text { for } x \notin F, \ell \in W^{\perp}-\operatorname{ri}\left[C(x, x F)^{*}\right] \\ 0 & \text { for } x \notin F, \ell \notin W^{\perp}-\operatorname{ri}\left[C(x, x F)^{*}\right]\end{cases}
$$

(ii) If $F$ has a half-line direct sum decomposition $F=M \oplus W \oplus L$ in (4), then

$$
\phi\left(H_{F}, x, \ell\right)= \begin{cases}(-1)^{\operatorname{dim} W} & \text { for } x \in F, \ell \in \operatorname{ri}(\operatorname{rec} F)^{*} \\ 0 & \text { for } x \in F, \ell \notin \operatorname{ri}(\operatorname{rec} F)^{*} \\ (-1)^{\operatorname{dim} W} & \text { for } x \notin F, \ell \in \operatorname{ri}(\operatorname{rec} F)^{*}-\operatorname{ri}\left[C(x, x F)^{*}\right] \\ 0 & \text { for } x \notin F, \ell \notin \operatorname{ri}(\operatorname{rec} F)^{*}-\operatorname{ri}\left[C(x, x F)^{*}\right]\end{cases}
$$

(iii) For the relatively open convex set $U$,

$$
\phi\left(H_{U}, x, \ell\right)= \begin{cases}(-1)^{\operatorname{dim} U} & \text { for } x \in U, \ell \in \angle U\rangle^{\perp} \\ 0 & \text { for } x \in U, \ell \notin\langle U\rangle^{\perp} \\ (-1)^{\operatorname{dim} U} & \text { for } x \notin U, \ell \in-C(x, x U)^{*} \\ 0 & \text { for } x \notin U, \ell \notin-C(x, x U)^{*}\end{cases}
$$

where $U^{\perp}$ denotes the subspace perpendicular to the affine space spanned by $U$.

Proof. (i) For $x \in F$, by definition of support functions we have $\ell(x) \leq h_{F}^{+}(\ell)$ for all $\ell \in \mathbf{V}^{*}$. Then $\phi\left(H_{F}, x, \ell\right)=(-1)^{\operatorname{dim} W}$ for $\ell \in W^{\perp}$ and $\phi\left(H_{F}, x, \ell\right)=0$ for $\ell \notin W^{\perp}$ by (i) of Lemma 5.3. Now for $x \notin F$, note that $C(x, x F)^{*}$ is contained in $W^{\perp}$. Let $\ell \in$ $W^{\perp}$. Then $\ell(x)>h_{F}^{+}(\ell)$ if and only if $\ell \in \operatorname{ri}\left[C\left(x, x F^{\prime}\right)^{*}\right]$. So $\phi\left(H_{F}, x, \ell\right)=(-1)^{\operatorname{dim} W}$ for $\ell \in W^{\perp}-\operatorname{ri}\left[C(x, x F)^{*}\right]$ and $\phi\left(H_{F}, x, \ell\right)=0$ for $\ell \notin W^{\perp}-\operatorname{ri}\left[C(x, x F)^{*}\right]$ by (i) of Lemma 5.3.

(ii) For $x \in F$, since $-\infty<\ell(x) \leq h_{F}^{+}(\ell)$ for all $\ell \in \mathbf{V}^{*}$, the formula is trivial by (ii) of Lemma 5.3. For $x \notin F$, note that $F \subset x+C(x, x F)$, then $\operatorname{rec} F \subset$ $\operatorname{rec}[x+C(x, x F)]=\operatorname{cl}[C(x, x F)]$. Clearly, $C(x, x F)^{*} \subset(\operatorname{rec} F)^{*}$, thus ri $\left[C(x, x F)^{*}\right] \subset$ ri $(\operatorname{rec} F)^{*}$. We now claim that $\phi\left(H_{F}, x, \ell\right) \neq 0$ for $\ell \in \operatorname{ri}(\operatorname{rec} F)^{*}-\operatorname{ri}\left[C(x, x F)^{*}\right]$. Let $G=F \cap W^{\perp}=M \oplus L$ and let $y$ be the image of $x$ under the orthogonal projection from $\mathrm{V}$ to $W^{\perp}$. Then $y \notin G$ and $\mathrm{cl}[C(y, y G)]$ is a pointed cone. In the subspace $W^{\perp}$, since $\operatorname{cl} C(y, r G)$ is a pointed cone with the apex $\{o\}, \ell \in \operatorname{ri}\left[C(y, y G)^{*}\right]$ if and only if $\ell^{\perp} \cap \operatorname{cl}[C(y, y * G)]=\{o\}$. Since $y \notin G$, this is equivalent to say that $h_{G}^{+}(\ell)<\ell(y)$. Note that $h_{F}^{+}(\ell)=h_{G}^{+}(\ell)$ and $\ell(x)=\ell(y)$, then $\ell \in \operatorname{ri}\left[C(x, x F)^{*}\right]$ if and only if $h_{F}^{+}(\ell)<\ell(x)$. By (ii) of Lemma 5.3, this shows that $\phi\left(H_{F}, x, \ell\right)=(-1)^{\operatorname{dim} W}$ if $\ell \in \operatorname{ri}(\operatorname{rec} F)^{*}-\operatorname{ri}\left[C(x, x F)^{*}\right]$. If $\ell \notin \operatorname{ri}\left(\operatorname{rec} F^{\prime}\right)^{*}-\operatorname{ri}\left[C(x, x F)^{*}\right]$, then either $\ell \notin \operatorname{ri}(\operatorname{rec} F)^{*}$ or $\ell \in \operatorname{ri}\left[C(x, x F)^{*}\right]$. In both cases, $\phi\left(H_{F}, x, \ell\right)=0$ by (ii) of Lemma 5.3.

(iii) For $x \in U$, if $\ell \in\langle U\rangle^{\perp}$, obviously, $\ell(U)=\left\{h_{U}^{-}(\ell)\right\}$, so $\phi\left(H_{U}, x, \ell\right)=(-1)^{\operatorname{dim} U}$ by (iii) of Lemma 5.3. If $\ell \notin\langle U\rangle^{\perp}$, obviously, $-\infty=h_{U}^{-}(\ell)<\ell(x)$, so $\phi\left(H_{U}, x, \ell\right)=0$. Now for $x \notin U$, if $\ell \in-C(x, x U)^{*}$, then $\ell(x) \leq h_{U}^{-}(\ell)$, so $\phi\left(1_{U}, x, \ell\right)=(-1)^{\operatorname{dim} U}$. If $\ell \notin-C(x, x U)^{*}$, then $\left(x+\ell^{\perp}\right) \cap U$ is non-empty and $\left(x+\ell^{\perp}\right) \cap U \neq U$. This shows that $h_{U}^{-}(\ell)<\ell(x)$. Therefore $\phi\left(H_{U}, x, \ell\right)=0$.

Theorem 5.5. The map $H: \mathbf{R}(\mathbf{V}, \mathcal{C} \cup \mathcal{O}) \longrightarrow F\left(\mathbf{V}^{*}, \mathbf{R}[\overline{\mathbf{R}}]\right)$, defined by $H(f)=$ $H_{f}$, is an injective linear map. Moreover, $H$ is an algebra homomorphism when restricted to $\mathbf{R}(\mathbf{V}, \mathcal{C})$ and $\mathbf{R}(\mathbf{V}, \mathcal{O})$. 
Proof. The linearity of $H$ is obvious. We want to claim that any convex chain $f \in \mathbf{R}(\mathbf{V}, \mathcal{C} \cup \mathcal{O})$ can be recovered from its support function $H_{f}$ as follows:

$$
f(x)=(-1)^{\operatorname{dim} \mathbf{V}} \chi\left[\phi\left(H_{f}, x\right)\right], x \in \mathbf{V} .
$$

To this end, we only need to verify that it is true for any closed convex set $F$ and any relatively open convex set $U$.

(i) If $F$ has a direct sum decomposition $F=K \oplus W$ in (4), then

$$
\chi\left[\phi\left(H_{F}, x\right)\right]= \begin{cases}(-1)^{\operatorname{dim} W} \chi\left(W^{\perp}\right)=(-1)^{\operatorname{dim} \mathrm{V}} & \text { for } x \in F \\ (-1)^{\operatorname{dim} W}\left(\chi\left(W^{\perp}\right)-\chi\left[\operatorname{ri} C(x, x F)^{*}\right]\right)=0 & \text { for } x \notin F\end{cases}
$$

since ri $\left[C(x, x F)^{*}\right]$ is a relatively open convex set of $\operatorname{dimension} \operatorname{dim} W^{\perp}$.

(ii) If $F$ has a half-line direct sum decomposition $F=M \oplus W \oplus L$, then

$$
\chi\left[\phi\left(H_{F}, x\right)\right]= \begin{cases}(-1)^{\operatorname{dim} W} \chi\left[\operatorname{ri}(\operatorname{rec} F)^{*}\right]=(-1)^{\operatorname{dim} \mathrm{V}} & \text { for } x \in F \\ (-1)^{\operatorname{dim} W}\left(\chi\left[\operatorname{ri}(\operatorname{rec} F)^{*}\right]-\chi\left[\operatorname{ri} C(x, x F)^{*}\right]\right)=0 & \text { for } x \notin F\end{cases}
$$

since $\mathrm{ri}(\operatorname{rec} F)^{*}$ and $\mathrm{ri}\left[C(x, x F)^{*}\right]$ are both relatively open convex sets of dimension $\operatorname{dim} W^{\perp}$.

(iii) For the relatively open convex set $U$, we have

$$
\chi\left[\phi\left(H_{U}, x\right)\right]= \begin{cases}(-1)^{\operatorname{dim} U} \chi\left(U^{\perp}\right)=(-1)^{\operatorname{dim} \mathbf{V}} & \text { for } x \in U \\ (-1)^{\operatorname{dim} U} \chi\left[-C(x, x U)^{*}\right]=0 & \text { for } x \notin U\end{cases}
$$

since $C(x, x U)^{*}$ is a closed convex cone and the Euler characteristic of any closed convex cone is always zero.

From the above observations we conclude that for $f \in \mathbf{R}(\mathbf{V}, \mathcal{C} \cup \mathcal{O})$,

$$
f(x)=(-1)^{\operatorname{dim} \mathbf{V}} \chi\left[\phi\left(H_{f}, x\right)\right], x \in \mathbf{V} .
$$

Since $\partial^{+}$is an algebra homomorphism from $\mathbf{R}(\overline{\mathbf{R}}, \mathcal{C} \cup \mathcal{O})$ to $\mathbf{R}[\overline{\mathbf{R}}]$ and $\ell_{*}^{\chi}$ is an algebra homomorphism from both $(\mathbf{V}, \mathcal{C})$ and $\mathbf{R}(\mathbf{V}, \mathcal{O})$ to $\mathbf{R}(\overline{\mathbf{R}}, \mathcal{C} \cup \mathcal{O})$, it follows that $\partial^{+} \ell_{*}^{\chi}$ is also an algebra homomorphism from $\mathbf{R}(\mathbf{V}, \mathcal{C})$ and $\mathbf{R}(\mathbf{V}, \mathcal{O})$ to $\mathbf{R}[\overline{\mathbf{R}}]$ for all $\ell \in \mathbf{V}^{*}$. Thus for any $f$ and $g$, both in $\mathbf{R}(\mathbf{V}, \mathcal{C})$ or both in $\mathbf{R}(\mathbf{V}, \mathcal{O})$, we have

$$
H_{f * g}=\partial^{+} \ell_{*}^{\chi}(f * g)=\partial^{+} \ell_{*}^{\chi}(f) * \partial^{+} \ell_{*}^{\chi}(g)=H_{f} * H_{g} .
$$

There are other embeddings for $\mathbf{R}(\mathbf{V}, \mathcal{C} \cup \mathcal{O})$ such as $\partial^{+} \ell_{*}^{\bar{\chi}}, \partial^{-} \ell_{*}^{\chi}$, and $\partial^{-} \ell_{*}^{\bar{\chi}}$. These induce injective algebra homomorphisms from $\mathbf{R}(\mathbf{V}, \mathcal{C})$ and $\mathbf{R}(\mathbf{V}, \mathcal{O})$ to $F\left(\mathbf{V}^{*}, \mathbf{R}[\overline{\mathbf{R}}]\right)$. If we only consider $H$ on $\mathbf{R}\left(\mathbf{V}, \mathcal{C}_{b} \cup \mathcal{O}_{b}\right)$, then $\partial^{+} \ell_{*}^{\chi}=\partial^{+} \ell_{*}^{\bar{\chi}}$ and $\partial^{-} \ell_{*}^{\chi}=\partial^{-} \ell_{*}^{\bar{\chi}}$, and we write $\partial^{+} \ell_{*}$ to denote $\partial^{+} \ell_{*}^{\chi}$ and $\partial^{+} \ell_{*}^{\bar{\chi}}$.

6. Multiplicative Units. We have pointed out that there is difficulty to define Minkowski multiplication for indicator functions of a closed convex set and a relatively open convex set. However, Theorem 5.5 enables us to define the multiplication through the embedding $H$.

DEFinition 6.1. The Minkowski algebra of convex sets is the smallest subalgebra $M\left(\mathbf{V}^{*}, \mathcal{C} \cup \mathcal{O}\right)$ of $F\left(\mathbf{V}^{*}, \mathbf{R}[\overline{\mathbf{R}}]\right)$, generated by the images of $\mathbf{R}(\mathbf{V}, \mathcal{C})$ and $\mathbf{R}(\mathbf{V}, \mathcal{O})$ under the embedding $H$ in Theorem 5.5. 
From now on, every multivalued function with multiplicities $f \in M\left(\mathbf{V}^{*}, \mathcal{C} \cup \mathcal{O}\right)$ can be written as

$$
f=\sum_{i} a_{i}(-1)^{\operatorname{dim} U_{i}} 1_{F_{i}} * \mathrm{~L}_{U_{i}}
$$

with some closed convex sets $F_{i}$ and relatively open convex sets $U_{i}$, and we write $f$ in this way without interpretation. Notice that $\chi(f): V^{*} \longrightarrow \mathbf{R}$, defined by $\chi(f)(\ell)=\chi\left[H_{f}(\ell)\right], \ell \in \mathbf{V}^{*}$, is a constant map, since $\chi\left[H_{f}(\ell)\right]=\chi(f)$ by Lemma 5.3.

Let $\phi, \psi \in M\left(\mathbf{V}^{*}, \mathcal{C} \cup \mathcal{O}\right)$ with $\chi(\phi)=\chi(\psi)=1$. 'Then for each $\ell \in \mathbf{V}^{*}, \phi$ and $\psi$ can be written respectively as

$$
\phi(\ell)=\sum a_{i}(\ell) 1_{\left\{p_{i}(\ell)\right\}} \text { and } \psi(\ell)=\sum b_{j}(\ell) 1_{\left\{q_{j}(\ell)\right\}},
$$

where $a_{i}(\ell), b_{j}(\ell)$ are non-zero numbers, and $\sum_{i} a_{i}(\ell)=\sum_{j} b_{j}(\ell)=1$. If $\phi$ and $\psi$ are the inverses of each other, then

$$
\begin{aligned}
\phi(\ell) * \psi(\ell) & =\sum a_{i}(\ell) b_{j}(\ell) 1_{\left\{p_{i}(\ell)\right\}} * 1_{\left\{q_{j}(\ell)\right\}} \\
& =\sum a_{i}(\ell) b_{j}(\ell) 1_{\left\{p_{i}(\ell)+q_{j}(\ell)\right\}}=1_{\{o\}} .
\end{aligned}
$$

Without loss of generality we may assume that $p_{1}<p_{2}<\cdots<p_{m}$ and $q_{1}<q_{2}<$ $\cdots<q_{n}$. Then $p_{1}+q_{1}<p_{i}+q_{j}<p_{m}+q_{n}$ if $(i, j)$ is neither $(1,1)$ nor $(m, n)$. Suppose $p_{1}+q_{1} \neq o$, then $a_{1} b_{1}=0$ and it forces that $a_{1}=0$ or $b_{1}=0$, a contradiction, so $p_{1}+q_{1}=o$. Similarly, $p_{m}+q_{n}=o$. This implies that $p_{i}+q_{j}=o$ for all $i$ and $j$. Thus $\phi$ must take the form $\phi(\ell)=1_{\{p(\ell)\}}$ and its inverse $\psi(\ell)=1_{\{-p(\ell)\}}$. This means that each invertible element of $M\left(\mathbf{V}^{*}, \mathcal{C} \cup \mathcal{O}\right)$ can be identified to a function $p$ on $\mathbf{V}^{*}$.

It is obvious from this observation that the indicator function of either a compact convex set or a bounded relatively open convex set is invertible. In fact, if $K$ is a compact convex set and $U$ a bounded relatively open convex set, then their inverses are given by

$$
\begin{aligned}
& \left(1_{K}\right)^{-1}=(-1)^{\operatorname{dim} K} 1_{- \text {ri } K}, \\
& \left(1_{U}\right)^{-1}=(-1)^{\operatorname{dim} U_{-\operatorname{cl} U} .}
\end{aligned}
$$

However, the indicator functions of unbounded closed convex sets and unbounded relatively open convex sets are not invertible. We now classify the group of invertible elements of the Minkowski algebra of convex sets. For simplicity, we only consider the invertible elements of $M\left(\mathbf{V}, \mathcal{C}_{b} \cup \mathcal{O}_{b}\right)$. A general element $f$ of $M\left(\mathbf{V}^{*}, \mathcal{C}_{b} \cup \mathcal{O}_{b}\right)$ can be written as

$$
f=\sum_{i=1}^{m} c_{i}(-1)^{\operatorname{dim} U_{i}} 1_{K_{i}} * 1_{U_{i}}
$$

where $K_{i}(1 \leq i \leq n)$ are compact convex sets and $U_{i}(1 \leq i \leq n)$ are bounded relatively open convex sets. If $f$ is invertible and $\chi(f)=\sum_{i=1}^{m} c_{i}=1$, then

$$
H_{f}(\ell)=\sum_{i=1}^{m} c_{i} 1_{\left\{h_{K_{i}}^{+}(\ell)+h_{U_{i}}^{-}(\ell)\right\}}=1_{\{p(\ell)\}}, \quad \forall \ell \in \mathbf{V}^{*}
$$


It has been shown [15] [18] that when $K_{i}$ are polytopes and $U_{i}$ are relatively open polytopes, the function $p(\ell)$ is actually a difference of the support functions of two polytopes. We suspect that the similar property for convex bodies is also true.

Conjecture 6.2. If $f$ is invertible in $M\left(\mathbf{V}^{*}, \mathcal{C}_{b} \cup \mathcal{O}_{b}\right)$ and $\chi(f)=1$, then there exist convex bodies $K$ and a relatively open convex set $U$ such that

$$
f=(-1)^{\operatorname{dim} U} 1_{K} * 1_{U}
$$

or equivalently, $p=h_{K}^{+}-h_{-\bar{U}}^{+}$when we write $f(\ell)=1_{\{p(\ell)\}}$. units.

By definition of $H$, we have the following relation about support functions and

THEOREM 6.3. The vector space spanned by the support functions of compact convex sets is isomorphic to a subgroup of units of the Minkowski algebra $M\left(\mathbf{V}^{*}, \mathcal{C} \cup \mathcal{O}\right)$ with the Euler characteristic equal to +1 , and the isomorphism is given by

$$
h_{K}^{+}-h_{-\bar{U}}^{+} \mapsto(-1)^{\operatorname{dim} U} 1_{K} * 1_{U}
$$

where $K$ is a compact convex set and $U$ a bounded relatively open convex set. In particular, $1_{t K}(t \geq 0)$ and $(-1)^{\operatorname{dim} K} 1_{t r i K}(t<0)$ form a one-parameter subgroup of the multiplicative units.

Let $\phi$ be a linear map from a vector space $\mathbf{V}$ to a vector space $\mathbf{W}$ of finite dimension. We can define a map $\phi_{*}^{\chi}: M\left(\mathbf{V}^{*}, \mathcal{C} \cup \mathcal{O}\right) \longrightarrow M\left(\mathbf{W}^{*}, \mathcal{C} \cup \mathcal{O}\right)$ by

$$
\phi_{*}^{\chi}(f)(\xi)=f(\xi \phi), \quad \forall f \in M\left(\mathbf{V}^{*}, \mathcal{C} \cup \mathcal{O}\right), \quad \xi \in \mathbf{W}^{*} \text {. }
$$

THEOREM 6.4. Let $\phi: \mathbf{V} \longrightarrow W$ be a linear map between the finite dimensional real vector spaces. Then $\phi_{*}^{\chi}$ is an algebra homomorphism from $M\left(\mathbf{V}^{*}, \mathcal{C} \cup \mathcal{O}\right)$ to $M\left(\mathbf{W}^{*}, \mathcal{C} \cup \mathcal{O}\right)$. Moreover, the following diagram is commutative.

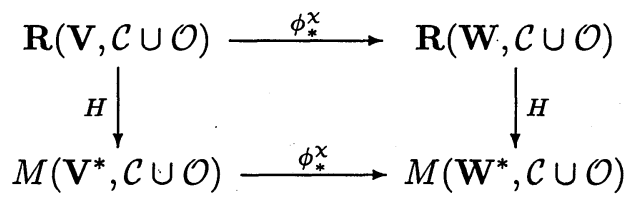

Proof. Given functions $f$ and $g$ in $M\left(\mathbf{V}^{*}, \mathcal{C} \cup \mathcal{O}\right)$, for any $\xi \in \mathbf{W}^{*}$, we have

$$
\begin{aligned}
\phi_{*}^{\chi}(f * g)(\xi) & =(f * g)(\xi \phi)=f(\xi \phi) * g(\xi \phi) \\
& =\phi_{*}^{\chi}(f)(\xi) * \phi_{*}^{\chi}(g)(\xi)=\left[\phi_{*}^{\chi}(f) * \phi_{*}^{\chi}(g)\right](\xi)
\end{aligned}
$$

By Proposition 3.6 we have

$$
\begin{aligned}
H \phi_{*}^{\chi}(f)(\xi) & =\partial^{+} \xi_{*}^{\chi}\left(\phi_{*}^{\chi} f\right)=\partial^{+}(\xi \phi)_{*}^{\chi}(f) \\
& =H(f)(\xi \phi)=\phi_{*}^{\chi}(H(f))(\xi)
\end{aligned}
$$

This shows that $\phi_{*}^{\chi}$ is an algebra homomorphism and the diagram is commutative. 
7. Euler-Radon Transform. Let $\mathrm{V}$ be a finite dimensional real vector space. For $k=0,1, \cdots, \operatorname{dim} \mathbf{V}$, the affine Grassmannian of $k$-flats of $\mathbf{V}$ is denoted by $A(\mathbf{V}, k)$ and the Grassmannian of $k$-subspaces of $\mathbf{V}$ is denoted by $G(\mathbf{V}, k)$. For a convex subset $E$ of $\mathbf{V}$, we define

$$
\begin{aligned}
& A(E, k)=\{H \in A(\mathbf{V}, k) \mid H \cap E \neq \emptyset\}, \\
& G(E, k)=\{H \in G(\mathbf{V}, k) \mid H \cap E \neq \emptyset\} .
\end{aligned}
$$

For any function $f$ in $\mathbf{R}(\mathbf{V}, \mathcal{C} \cup \mathcal{O})$, the Euler-Radon transform of $f$ is a function $\tilde{f}$ on $A(V)=\bigcup_{k=0}^{\infty} A(\mathbf{V}, k)$, defined by

$$
\tilde{f}(H)=\int_{H} f(x) \mathrm{d} \chi(x) .
$$

Let $(\tilde{f})_{1}$ denote the Euler-Radon transform of $f$ on $A(\mathbf{V}, 1)$. Notice that $\tilde{f}$ induces to a function $(\tilde{f})_{\mathbf{V}}$ on $\mathbf{V}$, defined by $(\tilde{f})_{\mathbf{V}}(v)=\tilde{f}(\mathbf{R} v)$ for $v \neq o$ and $(\tilde{f})_{\mathbf{V}}(o)=f(o)$. For a non-empty closed convex set $F$ and a non-empty relatively open convex set $U$, it can be verified that

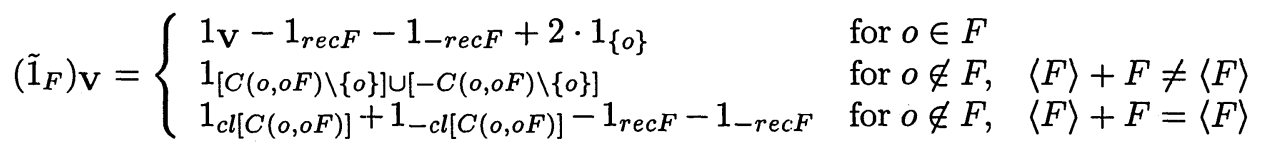

and

$$
\left(\tilde{1}_{U}\right)_{\mathbf{V}}=\left\{\begin{array}{lll}
1_{\mathbf{V}}-2 \cdot 1_{\langle U\rangle}+2 \cdot 1_{\{o\}} & \text { for } o \in U \\
1_{[C(o, o U) \backslash\{o\}] \cup[-C(o, o U) \backslash\{o\}]} & \text { for } o \notin U, & \langle U\rangle+U \neq\langle U\rangle \\
-1_{[C(o, o U) \backslash\{o\}] \cup[-C(o, o U) \backslash\{o\}]} & \text { for } o \notin U, & \langle U\rangle+U=\langle U\rangle .
\end{array}\right.
$$

Notice that $\operatorname{rec} F$ and $F$ are the same type of closed convex sets, and hence we have $\chi(\operatorname{rec} F)=\chi(F)$. For $o \notin U$, since $C(o, o U) \backslash\{o\}$ is a relatively open convex set of dimension $\operatorname{dim} U$, then $C(o, o U) \backslash\{o\}$ and $-C(o, o U) \backslash\{o\}$ are disjoint and $\chi[C(o, o U) \backslash\{o\}]=(-1)^{\operatorname{dim} U}$. Thus

$$
\begin{gathered}
\chi\left(\left(\tilde{1}_{F}\right)_{\mathbf{V}}\right)= \begin{cases}(-1)^{\operatorname{dim} \mathbf{V}}-2 \chi(F)+2 & \text { for } o \in F, \\
-2 \chi(F) & \text { for } o \notin F .\end{cases} \\
\chi\left(\left(\tilde{1}_{U}\right)_{\mathbf{V}}\right)= \begin{cases}(-1)^{\operatorname{dim} \mathbf{V}}-2(-1)^{\operatorname{dim} U}+2 & \text { for } o \in U \\
-2(-1)^{\operatorname{dim} U} & \text { for } o \notin U .\end{cases}
\end{gathered}
$$

By linearity of $\chi$ the value of $f$ at the origin can be recovered by

$$
f(o)=\left[2 \chi(f)+\chi\left((\tilde{f})_{\mathbf{v}}\right)\right] /\left[2+(-1)^{\operatorname{dim} \mathbf{v}}\right] .
$$

Let $t_{x}$ be the translation by $x$, that is, $t_{x}(v)=v+x$ for all $v \in \mathbf{V}$. Then the value $f(x)$ can be recovered by

$$
\begin{aligned}
f(x) & =\left[2 \chi\left(f t_{x}\right)+\chi\left(\left(f \tilde{t}_{x}\right) \mathbf{v}\right)\right] /\left[2+(-1)^{\operatorname{dim} \mathbf{v}}\right] \\
& =\left[2 \chi(f)+\chi\left(\left(f \tilde{t}_{x}\right)_{\mathbf{v}}\right)\right] /\left[2+(-1)^{\operatorname{dim} \mathbf{v}}\right]
\end{aligned}
$$


Now if $(\tilde{f})_{1}=0$, then $(\tilde{f})_{\mathbf{V}}=0$ except at $v=o$, where $(\tilde{f})_{\mathbf{V}}(o)=f(o)$, and this implies that

$$
\left[1+(-1)^{\operatorname{dim} V}\right] f(x)=2 \chi(f) .
$$

For even $\operatorname{dim} \mathbf{V}$, we have $f(x)=\chi(f)$, showing that $f$ is a constant function. So $(\tilde{f})_{1}=-\chi(f)$, whence $f=0$. For odd $\operatorname{dim} \mathbf{V}$, then for any 2-dimensional plane $H$, $\left(\left.f\right|_{H}\right)_{1}=0$, which implies $\left.f\right|_{H}=0$, whence $f=0$. Thus we have proved the following theorem.

THEOREM 7.1. The linear transformation $(\tilde{f})_{1}$ is injective.

We can also identify the Euler-Radon transform on the co-dimension one subspaces to a transform $(\tilde{f})_{\mathbf{V}}^{*}$ on the dual space $\mathbf{V}^{*}$, defined by

$$
(\tilde{f})_{\mathbf{V}}^{*}(\xi)=\tilde{f}\left(\xi^{-1}(0)\right), \quad \xi \in \mathbf{V}^{*} .
$$

If $F=K \oplus W$ is a closed convex set of type I, it can be easily shown that

$$
\left(\tilde{1}_{F}\right)_{\mathbf{V}^{*}}= \begin{cases}2(-1)^{\operatorname{dim} W} 1_{W^{\perp}}-(-1)^{\operatorname{dim} W} 1_{\mathbf{V}^{*}} & \text { for } o \in F \\ (-1)^{\operatorname{dim} W}\left[2 \cdot 1_{W^{\perp}}-1_{\mathbf{V}^{*}}-1_{r i\left[C(o, o F)^{*}\right]}-1_{-r i\left[C(o, o F)^{*}\right]}\right] & \text { for } o \notin F .\end{cases}
$$

If $F=M \oplus W \oplus L$ is a closed convex set of type II, it can be similarly shown that

$$
\left(\tilde{1}_{F}\right)_{\mathrm{V}^{*}}= \begin{cases}(-1)^{\operatorname{dim} W} 1_{r i\left[(r e c F)^{*}\right]}+(-1)^{\operatorname{dim} W} 1_{-r i\left[(r e c F)^{*}\right]} & \text { for } o \in F \\ 1_{r i\left[(r e c F)^{*}\right]}+1_{-r i\left[(r e c F)^{*}\right]}-1_{r i\left[C(o, o F)^{*}\right]}-1_{-r i\left[C(o, o F)^{*}\right]} & \text { for } o \notin F .\end{cases}
$$

For a non-empty relatively open convex set $U$, it can be analogously verified that

$$
\left(\tilde{1}_{U}\right)_{\mathbf{V}^{*}}= \begin{cases}(-1)^{\operatorname{dim} U}\left[2 \cdot 1_{\langle U\rangle^{\perp}}-1_{\mathbf{V}^{*}}\right] & \text { for } o \in U \\ (-1)^{\operatorname{dim} U}\left[1_{C(o, o U)^{*}}+1_{C(o, o U)^{*}}-1_{\mathbf{V}^{*}}\right] & \text { for } o \notin U\end{cases}
$$

By the linearity of the Euler-Radon transform, the value of $f$ at the origin can be similarly recovered by

$$
f(o)=\left[\chi(f)+(-1)^{\operatorname{dim} \mathbf{v}} \chi\left((\tilde{f}) \mathbf{V}^{*}\right)\right] / 2 .
$$

Let $t_{x}$ be the translation by $x$ again, we then have

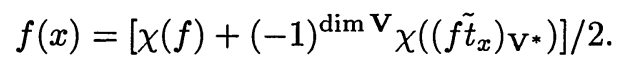

Notice that $\left(\tilde{t}_{x}\right)_{\mathbf{V}^{*}}(\xi)=\chi\left(f \cdot 1_{\xi^{-1}(0)+x}\right)$. Now if the Euler-Radon transform $\tilde{f}=0$ for all co-dimension one affine planes, then $\left(\tilde{f}_{x}\right) \mathbf{V}^{*}=0$ for all co-dimension one subspaces and $\chi\left(\left(f \tilde{t}_{x}\right)_{\mathbf{V}^{*}}\right)=0$. This means that $f(x)=\chi(f) / 2$ is a constant function. Thus $\chi(f)=\chi(f)(-1)^{\operatorname{dim} \mathrm{V}} / 2$, which implies $\chi(f)=0$, whence $f=0$.

Let $(\tilde{f})_{k}$ denote the Euler-Radon transform restricted to the affine Grassmannian $A(\mathbf{V}, k)$ of $k$-flats. It follows from the Fubini theorem for Euler integrals that if $(\tilde{f})_{k}=0$, then $(\tilde{f})_{\operatorname{dim} \mathbf{V}-1}=0$, whence $f=0$ by the above observation. This gives the following theorem.

THEOREM 7.2. For each $0 \leq k \leq \operatorname{dim} \mathbf{V}-1$, the Euler-Radon transform $(\tilde{f})_{k}$ is injective.

For each non-negative integer $k \leq n$ and a bounded closed convex set $K$ of $\mathbf{R}^{n}$, we denote by $I_{K}^{(k)}$ the indicator function of $A(K, k)$ on the affine Grassmannian $A(n, k)$ 
of $k$-flats. We shall consider the vector space $\mathbf{R}\left(A(n, k), A\left(\mathcal{C}_{b}, k\right)\right)$ generated by the indicator functions of members of $A\left(\mathcal{C}_{b}, k\right)=\left\{A(K, k) \mid K \in \mathcal{C}_{b}\right\}$, that is, the space of functions of the form

$$
f=a_{1} I_{K_{1}}^{(k)}+\cdots+a_{m} I_{K_{n}}^{(k)}
$$

where $K_{i}(1 \leq i \leq m)$ are bounded closed convex sets of $\mathbf{R}^{n}$ and $m$ is any positive integer.

An interesting problem of Klain and Rota on geometric probability [10] is to classify all linear relations in $\mathbf{R}\left(A(n, k), A\left(\mathcal{C}_{b}, k\right)\right)$, that is, the relations of the form

$$
\sum_{i=1}^{m} a_{i} I_{K_{i}}^{(k)}=0
$$

For $k=0$, the function $I_{K}^{(0)}$ for a compact convex set $K$ is the indicator function $1_{K}$. This special case was solved by Rota in an indirect way by introducing valuation rings of distributive lattices [21]. Since the class $U\left(\mathcal{C}_{b}\right)$ of finite unions of compact convex sets is a distributive lattice of sets, of which the union is the joint operation and the intersection is the meet operation, and the valuation ring of $U\left(\mathcal{C}_{b}\right)$ is canonically isomorphic to $\mathbf{R}\left(\mathbf{V}, U\left(\mathcal{C}_{b}\right)\right)=\mathbf{R}\left(\mathbf{V}, \mathcal{C}_{b}\right)$. With this identification and the definition of the valuation ring, all linear relations in $\mathbf{R}\left(\mathbf{V}, \mathcal{C}_{b}\right)$ are generated by inclusion-exclusion formulas:

$$
1_{K_{1} \cup \cdots \cup K_{m}}=\sum_{j=1}^{m}(-1)^{j-1} \sum_{i_{1}<\cdots<i_{j}} 1_{K_{i_{1}} \cap \cdots \cap K_{i_{j}}},
$$

where $K_{i}(1 \leq i \leq m)$ and $K_{1} \cup \cdots \cup K_{m}$ are compact convex sets.

The syzygy problem of Rota on $A(n, k)$ is to classify all linear relations on $\mathbf{R}\left(A(n, k), A\left(\mathcal{C}_{b}, k\right)\right)(0 \leq k \leq n)$. For $k=n$, it is trivial. For $k=1$ and 2 , the problem was thought probably solved by Ambartzumian. However, the problem was considered particularly difficult for $k \geq 3$ in [10]. Nevertheless, we can easily solve this problem in all dimensions by the Euler-Radon transform and Theorem 7.2.

THEOREM 7.3. All linear relations in $\mathbf{R}\left(A(n, k), A\left(\mathcal{C}_{b}, k\right)\right)(0 \leq k \leq n)$ are generated by the inclusion-exclusion formulas:

$$
I_{K_{1} \cup \cdots \cup K_{m}}^{(k)}=\sum_{j=1}^{m}(-1)^{j-1} \sum_{i_{1}<\cdots<i_{j}} I_{K_{i_{1}} \cap \cdots \cap K_{i_{j}}}^{(k)},
$$

where $K_{i}(1 \leq i \leq m)$ and $K_{1} \cup \cdots \cup K_{m}$ are compact convex sets.

Proof. Let $K$ and $L$ be compact convex sets of $\mathbf{V}$. It is obvious that

$$
A(K \cup L, k)=A(K, k) \cup A(L, k) .
$$

If $K \cup L$ is also convex, then a $k$-plane intersects both $K$ and $L$ if and only if the $k$-plane intersects $K \cap L$, that is,

$$
A(k, K \cap L)=A(k, K) \cap A(k, L) .
$$

So if $K \cup L$ is convex we have the inclusion-exclusion formula:

$$
I_{K}^{(k)}+I_{L}^{(k)}=I_{K \cup L}^{(k)}+I_{K \cap L}^{(k)} .
$$


This means that the linear relations in $\mathbf{R}\left(A(n, k), A\left(\mathcal{C}_{b}, k\right)\right)$ include all inclusionexclusion formulas.

Since the Euler-Radon transform is injective, a linear relation

$$
a_{1} I_{K_{1}}^{(k)}+\cdots+a_{m} I_{K_{m}}^{(k)}=0
$$

holds in $\mathbf{R}\left(A(n, k), A\left(\mathcal{C}_{b}, k\right)\right)$ if and only if the linear relation

$$
a_{1} 1_{K_{1}}+\cdots+a_{m} 1_{K_{m}}=0
$$

holds in $\mathbf{R}\left(\mathbf{R}^{n}, \mathcal{C}_{b}\right)$. Thus all linear relations in $\mathbf{R}\left(A(n, k), A\left(\mathcal{C}_{b}, k\right)\right)$ are generated by (28).

The syzygy problem can be posed on flag manifolds or more generally on partition varieties [6]. The discrete analog has been posted by Klain and Rota on a partially ordered set [10] and the inclusion-exclusion is replaced by the relation specified by the Möbius function [20]. The mixed volumes and invariant geometric measures can be similarly investigated on $M\left(\mathbf{V}^{*}, \mathbf{R}[\overline{\mathbf{R}}]\right)$ via Geissinger multiplications [5] [7] and Euler characteristics.

\section{REFERENCES}

[1] Ju. D. Burago and V. A. Zalgaller, Geometric Inequalities, Springer-Verlag, 1980.

[2] B. CHEN, The incidence algebra of polyhedra over the Minkowski algebra, Advances in Math., 118 (1996), pp. 337-365.

[3] B. CHEN, On the Euler characteristics of finite unions of convex sets, Discrete Comput. Geom., 10 (1993), pp. 79-93.

[4] B. CHEN, The Minkowski algebra of convex sets, MIT preprint, May 1992.

[5] B. CHEN, The mixed volumes and Geissinger multiplications of convex sets, Studies in Applied Math., 91 (1994), pp. 39-50.

[6] K. DING, Rook placements and cellular decomposition of partition varieties, to appear.

[7] L. GeIssinger, Valuations on distributive lattices I, II, III, Arch. Math., 24 (1973), pp. 230$239,337-345,475-481$.

[8] H. Groemer, Minkowski addition and mixed volumes, Geom. Dedicata, 6 (1977), pp. 141-163.

[9] H. HADWIGER, Vorlesungen über Inhalt, Oberflä̈che und Isoperimetrie., Springer, Berlin, 1957.

[10] D. A. KLAIN AND G.-C. Rota, Introduction to Geometric Probability, Cambridge Univ. Press, Lezioni Lincee, 1997.

[11] V. L. KLEE, Asymptotes and projections of convex bodies, Math. Scand., 8 (1960), pp. 356-362.

[12] V. L. KLEE, Semicontinuity of the face-function of a convex set, Commentarii Mathematici Helvetici, 46 (1971), pp. 1-12.

[13] J. LaWrence, Minkowski rings, Preprint, September 1985.

[14] P. MCMullen, The polytope algebra, Advances in Math., 78 (1989), pp. 76-130.

[15] R. MoRelli, A theory of polyhedra, Advances in Math., 97 (1993), pp. 1-73.

[16] T. ODA, Convex Bodies and Algebraic Geometry: an Introduction to the Theory of Toric Varieties, Springer-Verlag, 1982.

[17] K. PRZESLAWSKI, Linear algebra of convex sets and the Euler characteristic, Linear and Multilinear Algebra, 31 (1992), pp. 143-191.

[18] A. V. PUKhlikov AND A. G. KhOVANŚKII, Finitely additive measures of virtual polytopes, St. Petersburg Math. J., 4 (1993), pp. 337-356.

[19] R. Rockafellar, Convex Analysis, Princeton Univ. Press, 1970.

[20] G.-C. RotA, On the foundations of combinatorial theory I. Theory of Möbius functions, Z. Wahrscheinlichkeitstheorie, 2 (1964), pp. 340-368.

[21] G.-C. Rota, On the combinatorics of the Euler characteristic, Studies in Pure Mathematics (Papers presented to Richard Rado), pp. 221-233, Academic Press, London 1971.

[22] L. A. Santaló, Integral Geometry and Geometric Probability, Addison-Wesley Publishing Co., 1976.

[23] S. SchanUel, What is the Length of a Potato? An Introduction to Geometric Measure Theory, Lecture Notes in Mathematics, 1174, Springer, 1986. 
[24] S. Schanuel, Negative Sets have Euler Characteristic and Dimension, Lecture Notes in Mathematics, 1488, Springer, New York, 1991.

[25] R. SChneIder, Convex Bodies: The Brunn-Minkowski Theory, Cambridge Univ. Press, 1993. 